\title{
IMAGE OF SCHWARTZ SPACE UNDER SPECTRAL PROJECTION
}

\author{
JOYDIP JANA
}

\begin{abstract}
Let $X=G / K$ symmetric space of non compact type, where $G$ is a rank-one connected semisimple Lie group with finite center. We shall look at the transform $P_{\lambda} f(x)=f * \varphi_{\lambda}(x)$, where, $\lambda \in \mathbb{C}$ and $\varphi_{\lambda}$ is the elementary spherical function. We shall try to characterizes the image of the Schwartz spaces $S^{p}(X)$ where $0<p \leq 2$ under the above transform.
\end{abstract}

\section{Introduction}

Let $X$ be the Riemannian symmetric space realized as $G / K$ where $G$ is a connected, noncompact, real rank-one semi-simple Lie group with finite center. Let $\varphi_{\lambda}(\lambda \in \mathbb{C})$ be the elementary spherical functions of $G$. We denote $\mathcal{S}^{p}(X)(0<p \leq 2)$ for the $L^{p}$-Schwartz class functions on $X$. For $f \in \mathcal{S}^{p}(X)$ $(0<p \leq 2)$ (for the case $\left.1<p \leq 2, f \in L^{p}(X)\right)$ we consider the transform $f \mapsto P_{\lambda} f(x)=f * \varphi_{\lambda}(x)$ for each $\lambda$ in a suitable domain. The function $P_{\lambda} f$ is an eigenfunction of the Laplacian $\mathbf{L}$ of the group $G$, satisfying $\mathbf{L} P_{\lambda} f(x)=-\left(1+\lambda^{2}\right) P_{\lambda} f(x)$ and the transform $f \mapsto P_{\lambda} f$ is called the generalized spectral projection. Strichartz in his series of papers [Str88], [Str89], [Str91], [Str92] initiated the project of reviewing Harmonic Analysis in terms of the generalized spectral projection. Continuing this project Bray [Bra96] proved a spectral Paley-Wiener theorem for the symmetric space $X=G / K$. Ionescu [Ion00] characterized the image $P_{\lambda}\left(L^{2}(X)\right)$. Strichartz [Str89] determined the image of Euclidean Schwartz class functions under spectral projection.

The aim of this paper is to characterize the image of the $L^{p}$-Schwartz space $\mathcal{S}^{p}(X)(0<p \leq 2)$ under the transform $f \mapsto P_{\lambda} f$. For each $f \in \mathcal{S}^{p}(X), P_{\lambda} f$ is a function on $\mathfrak{a}_{\varepsilon}^{*} \times X$ where $\mathfrak{a}_{\varepsilon}^{*}=$ $\left\{\lambda \in \mathbb{C}|| \Im \lambda \mid<\varepsilon=\left(\frac{2}{p}-1\right)\right\}$.

The characterization of the image of $\mathcal{S}^{p}(X)$ under the generalized spectral projection is divided into two parts. In Section 3 we obtain some basic properties (necessary conditions) of the functions $P_{\lambda} f$ for $f \in \mathcal{S}^{p}(X)$. Sufficient conditions for a left-K-finite function $f(\lambda, x)$ on $\mathfrak{a}_{\varepsilon}^{*} \times X$ to be of the form $P_{\lambda} g(x)=f(\lambda, x)$ for some $g \in \mathcal{S}^{p}(X)$ are taken up in Section 4. Finally, in Section 5, we shall characterize the image of certain subspace of $L^{2}(X)$ under the above mentioned transform in the light of the inverse Paley-Wiener theorem due to Thangavelu [Tha07]. For this section we refer [GKÓ06a, GKÓ06b, GK02, KS04, KS05] and [Tha07].

\section{Preliminaries}

In this section we shall briefly recall some basic facts and results about noncompact Riemannian symmetric spaces realized as $X=G / K$ where $G$ be a connected noncompact semisimple Lie group with finite center and $K$ a maximal compact subgroup of $G$. Let $\mathfrak{k}$ be the Lie algebra of $K$. We fix an Iwasawa decomposition $G=K A N$ and let $\mathfrak{a}$ be the Lie algebra of the abelian subgroup $A$. In this discussion we shall mainly concentrate on the 'rank-one' Riemannian symmetric spaces so our associated semisimple Lie group $G$ will be of 'real rank-one'. Hence $A$ and $\mathfrak{a}$ both are of dimension one. Let $\mathfrak{a}^{*}$ be the real dual of $\mathfrak{a}$ and $\mathfrak{a}_{\mathbb{C}}^{*}$ be its complexification. The Killing form induces a positive definite form $\langle\cdot, \cdot\rangle$ on $\mathfrak{a}^{*} \times \mathfrak{a}^{*}$, let the bilinear extension of this form on $\mathfrak{a}_{\mathbb{C}}^{*} \times \mathfrak{a}_{\mathbb{C}}^{*}$ be denoted by $\langle\cdot, \cdot\rangle_{1}$. In the rank-one case the restricted roots for the adjoint action of $\mathfrak{a}$ on $\mathfrak{g}$ are of the form $\pm \gamma$ and (possibly) $\pm 2 \gamma$ with $\gamma \in \mathfrak{a}^{*}$. We denote $m_{\gamma}$ for the multiplicity of the root $\gamma$. Let $\rho \in \mathfrak{a}^{*}$ be the half sum of the positive roots. With a proper

Date: September 22, 2021.

Key words and phrases. Spectral projection, Helgason Fourier transform, Schwartz space, Complex crown.

The author is thankful to Prof. S. C. Bagchi and Rudra P. Sarkar of Indian Statistical Institute for their useful suggestions.

Mathematical Subject Classification: 43A80, 43A85, 43A90. 
normalization the linear functional $\rho$ can be identified with the constant function $\rho(X)=1$. The same normalization also identifies $A, \mathfrak{a}, \mathfrak{a}^{*}$ with $\mathbb{R}$ and $\mathfrak{a}_{\mathbb{C}}^{*}$ with $\mathbb{C}$. The Weyl group in our case $W=\{+1,-1\}$ acts on $A, \mathfrak{a}, \mathfrak{a}^{*}$ and $\mathfrak{a}_{\mathbb{C}}^{*}$ simply by multiplication. We denote $\mathfrak{a}^{+}$a cone in $\mathfrak{a}$ called the positive Weyl chamber. Let $\mathfrak{a}^{*+} \subset \mathfrak{a}^{*}$ be the cone dual to $\mathfrak{a}^{+}$. In the rank-one case both $\mathfrak{a}^{+}$and $\mathfrak{a}^{*+}$ essentially correspond to the set $\mathbb{R}^{+}$of all positive numbers. Let $\overline{\mathfrak{a}^{+}}$and $\overline{\mathfrak{a}^{*+}}$ respectively denote the closures of the cones $\mathfrak{a}^{+}$and $\mathfrak{a}^{*+}$ also let us denote $\overline{A^{+}}=\exp \overline{\mathfrak{a}^{+}}$. The group elements of $A$ will now be denoted by $a_{t}$ where $t \in \mathbb{R}$ and $\exp t=a_{t}$. Let $H: g=k a_{t} n \mapsto H(g)=t \in \mathfrak{a}$ be the Iwasawa-a-projection of $G$ in $\mathfrak{a}$ for the $K A N$ decomposition of the group.

The Cartan decomposition gives $G=K \overline{A^{+}} K$. It induces a diffeomorphism from $K / M \times A^{+} \times K$ (or $\left.K \times A^{+} \times M \backslash K\right)$ onto an open dense subset of $G$ where $M$ is the centralizer of $A$ in $K$ ( $M$ also normalizes $N)$. Let $x^{+}$be the $\overline{\mathfrak{a}^{+}}$projection of $x \in G$ for the Cartan decomposition $x=k_{1}\left(\exp x^{+}\right) k_{2}$ and we denote $|x|=\left\|x^{+}\right\|$. For all $x \in G$ the Iwasawa-a-projection $H(x)$ and the quantity $|x|$ are related by the inequality:

$$
\|H(x)\| \leq c|x|, x \in G \text {, where } c>0 \text { is a fixed constant. }
$$

We also note that in the symmetric space $X=G / K,|x|$ is the Riemannian distance of $x K$ from the coset $e K, e$ being the identity element of $G$.

The Haar measure corresponding to the Iwasawa- $K A N$ decomposition is given by

$$
\int_{G} f(x) d x=\text { const. } \int_{K} d k \int_{\mathfrak{a}^{+}} e^{2 t} d t \int_{N} f\left(k a_{t} n\right) d n,
$$

where the const stands for a normalizing constant. In the case of the Cartan decomposition the Haar measure on $G$ is given by

$$
\int_{G} f(x) d x=\text { const. } \int_{K} d k_{1} \int_{\mathfrak{a}^{+}} \Delta(t) d t \int_{K} f\left(k_{1} a_{t} k_{2}\right) d k_{2},
$$

where the density function $\Delta(t)$ has the estimate $\Delta(t)=O\left(e^{2 t}\right)$. A function $f$ will be called right- $K$ invariant if it satisfies $f(x k)=f(x)$ for all $x \in G$ and $k \in K$. A function on the symmetric space $X=G / K$ can also be considered as a right- $K$-invariant function on the group $G$. We denote $\mathcal{C}^{\infty}(G)$ for the set of all smooth functions on $G$. Let $\mathcal{U}(\mathfrak{g})$ be the 'universal enveloping algebra' over $\mathfrak{g}$ and $\Omega$ be the Casimir element of $\mathcal{U}(\mathfrak{g})$. The action of the Laplace-Beltrami operator $\mathbf{L}$ on $X$ is defined by the action of Casimir operator $\Omega$ : $\mathbf{L} f(x K)=f(x ; \Omega)$ for all $x \in G$. In our discussion the following eigenspaces of the operator $\mathbf{L}$

$$
\mathcal{E}_{\lambda}(X)=\left\{g \in \mathrm{C}^{\infty}(X) \mid \mathbf{L} g(x)=-\left(\lambda^{2}+1\right) g(x)\right\}, \text { for each } \lambda \in \mathbb{C}
$$

will be of main interest. For each $\lambda \in \mathbb{C}$, let $\varphi_{\lambda}$ be the elementary spherical function associated with $\lambda$. We recall that $\varphi_{\lambda}(\lambda \in \mathbb{C})$ is given by the following integral representations [GV88]:

$$
\varphi_{\lambda}(x)=\int_{K} e^{-(i \lambda+1) H\left(x^{-1} k\right)} d k=\int_{K} e^{(i \lambda-1) H(x k)} d k .
$$

We recollect some of the very basic properties of the elementary spherical functions, which will be used throughout.

Proposition 2.1. $\quad$ (i) The expression $\varphi_{\lambda}(x)$ is a bi-K-invariant $\mathrm{C}^{\infty}$ function in the $x$ variable and it is a $W$-invariant holomorphic function in $\lambda \in \mathbb{C}$.

(ii) For each $\lambda \in \mathbb{C}, x \mapsto \varphi_{\lambda}(x)$ is a joint eigenfunction of all the $G$-invariant differential operators on $G / K$; in particular for the Laplace-Beltrami operator we have:

$$
\boldsymbol{L} \varphi_{\lambda}(\cdot)=-\left(\lambda^{2}+1\right) \varphi_{\lambda}(\cdot), \quad \lambda \in \mathbb{C} .
$$

(iii) For each $\lambda \in \mathbb{C}$ and $x, y \in G$, the following property is referred to as the 'symmetric property of the elementary spherical functions'

$$
\varphi_{\lambda}\left(x^{-1} y\right)=\int_{K} e^{-(i \lambda+1) H\left(y^{-1} k^{-1}\right)} e^{(i \lambda-1) H\left(x^{-1} k^{-1}\right)} d k .
$$

(iv) For any given $\boldsymbol{D}, \boldsymbol{E} \in \mathcal{U}(\mathfrak{g})$, there exists a constant $c>0$ such that

$$
\left|\varphi_{\lambda}(\boldsymbol{D} ; x ; \boldsymbol{E})\right| \leq c(|\lambda|+1)^{\operatorname{deg} \boldsymbol{E}+\operatorname{deg} \boldsymbol{D}} \varphi_{i \Im \lambda}(x) \quad \text { for all } x \in G, \lambda \in \mathbb{C} .
$$


(v) Given any polynomial $P$ in the algebra $\mathbf{S}(\mathfrak{a})$ of symmetric polynomials on $\mathfrak{a}^{*}$, there exists a positive constant $c$ such that:

$$
\left|P\left(\frac{\partial}{\partial \lambda}\right) \varphi_{\lambda}(x)\right| \leq c(1+|x|)^{\operatorname{deg} P} \varphi_{i \Im \lambda}(x), \quad x \in G .
$$

(vi) For all $t$ and $\lambda$ in $\overline{\mathbb{R}^{+}}$we have:

$$
0<\varphi_{-i \lambda}\left(a_{t}\right) \leq e^{\lambda t} \varphi_{0}\left(a_{t}\right) .
$$

(vii) For all $x \in G$, we have $0<\varphi_{0}(x)=\varphi_{0}\left(x^{-1}\right) \leq 1$;

(viii) For all $t \in \overline{\mathbb{R}^{+}}$, we have the following two-side estimate of $\varphi_{0}$ :

$$
e^{-t} \leq \varphi_{0}\left(a_{t}\right) \leq c(1+t)^{a} e^{-t}
$$

where $c, a>0$ are group dependent constants;

Property (i) is a very basic fact which follows from the definition. For a proof one can see [GV88, Ch. 4]. Property (ii) was proved by Helgason [Hel00]. For (iii) we refer to [Hel94, Ch. III, Theorem 1.1]. The estimates (iv), (v), and (vi) follows from the results in [GV88, Sec. 4.6]. For a direct and a simple proof of (iv) and (v) one can see [Ank91, Proposition 3]. The estimate (vii) of $\varphi_{0}$ is due to Harish-Chandra. A proof of this can be found in [GV88, Theorem 4.6.4, Theorem 4.6.5]. We should note that a sharper two-sided estimate of $\varphi_{0}$ is given by Anker [Ank87].

Let $\delta$ be an unitary irreducible representation of $\mathrm{K}$ i.e $\delta \in \widehat{K}$ with $V_{\delta}$ (a finite dimensional vector space) the representation space. Let $\chi_{\delta}$ stand for the character of the representation $\delta$. Let $V_{\delta}^{M}$ be the subspace of $V_{\delta}$ fixed under $\left.\delta\right|_{M}$; i.e $V_{\delta}^{M}=\left\{v \in V_{\delta} \mid \delta(m) v=v \forall m \in M\right\}$. Kostant [Kos69] proved that for a rank-one group the dimension of $V_{\delta}^{M}$ is 0 or 1 . Let $\widehat{K}_{M}$ be the set of all equivalence classes of irreducible unitary representation $\delta$ of $\mathrm{K}$ for which $V_{\delta}^{M} \neq\{0\}$. For our result we choose $\delta \in \widehat{K}_{M}$.

As, $\delta \in \widehat{K}_{M}, \delta(k)$ is a unitary matrix of order $d_{\delta}$. So $\|\delta(k)\|_{\mathbf{2}}=d_{\delta}^{\frac{1}{2}}$ where, $\|\cdot\|_{\mathbf{2}}$ denotes the Hilbert Schmidt norm. One can associate a norm $|\delta|$ to each unitary irreducible representation $\delta$ of $K$ (for explicit constriction of this norm we refer [EK 76]). From Weyl's dimension formula we can choose an $r \in \mathbb{Z}^{+}$and a positive constant $c$ independent of $\delta$ such that

$$
\|\delta(k)\|_{2} \leq c(1+|\delta|)^{r}
$$

for all $k \in K$. Thus $d_{\delta} \leq c^{\prime}(1+|\delta|)^{2 r}$ clearly, again $c^{\prime}$ is independent of the chosen $\delta$. For any $f \in \mathrm{e}^{\infty}(X)$ we put:

$$
f^{\delta}(x)=d_{\delta} \int_{K} f(k x) \delta\left(k^{-1}\right) d k .
$$

Clearly, $f^{\delta}$ is a $\complement^{\infty} \operatorname{map}$ from $X$ to $\operatorname{Hom}\left(V_{\delta}, V_{\delta}\right)$ satisfying

$$
f^{\delta}(k x)=\delta(k) f^{\delta}(x), \text { for all } x \in X, k \in K .
$$

Any function satisfying the property (2.14) will be referred to as (a $d_{\delta} \times d_{\delta}$ matrix valued) left $\delta$-type function. For any function space $\mathcal{E}(X) \subseteq \mathcal{C}^{\infty}(X)$, we write $\mathcal{E}^{\delta}(X)=\left\{f^{\delta} \mid f \in \mathcal{E}(X)\right\}$. We shall denote by $\check{\delta}$ the contragradient representation of the representation $\delta \in \widehat{K}_{M}$. A function $f$ will be called a scalar valued left- $\delta$-type function if $f=d_{\delta} \chi_{\delta} * f$, where the operation $*$ is the convolution over $K$. For any class of scalar valued functions $\mathcal{G}(X)$ we shall denote $\mathcal{G}(\delta, X)=\left\{g \in \mathcal{G}(X) \mid g=d_{\delta} \chi_{\delta} * g\right\}$. Throughout our discussion we fix the notation $\mathcal{D}(X)$ for the subclass of functions in $\mathrm{e}^{\infty}(X)$ which are of compact support. The following theorem, due to Helgason, identifies the two classes $\mathcal{D}^{\delta}(X)$ and $\mathcal{D}(\check{\delta}, X)$ corresponding to each $\delta \in \widehat{K}_{M}$.

Theorem 2.2. [Helgason [Hel94, Ch.III, Proposition 5.10]]

The map $\mathcal{Q}: f \mapsto g, g(x)=\operatorname{tr}(f(x))(x \in X)$ is a homeomorphism from $\mathcal{D}^{\delta}(X)$ onto $\mathcal{D}(\check{\delta}, X)$ and its inverse is given by $g \mapsto f=g^{\delta}$. 
A consequence of the 'Peter-Weyl theorem' can be stated [Hel00, Ch.IV, Corollary 3.4] in the form that any $f \in \mathrm{e}^{\infty}(X)$ has the decomposition

$$
f(x)=\sum_{\delta \in \widehat{K}_{M}} \operatorname{tr}\left(f^{\delta}(x)\right),
$$

where the convergence is in the sense of uniform convergence on compacta. A function $f \in \mathcal{C}^{\infty}(X)$ is said to be left- $K$-finite if there exists a finite subset $\Gamma(f) \subset \widehat{K}_{M}$ (depending on the function $f$ ) such that $\operatorname{tr}\left(f^{\gamma}\right)=0$ for all $\gamma \in \widehat{K}_{M} \backslash \Gamma(f)$. For any class $\mathfrak{H}(X) \subseteq \mathcal{C}^{\infty}(X)$ of functions we shall denote by $\mathfrak{H}(X)_{K}$ the subclass consisting of the left- $K$-finite functions. Let $\Gamma$ be a fixed subset (finite or infinite) of $\widehat{K}_{M}$. Then we shall use the notation $\mathfrak{H}(\Gamma ; X)$ for the subclass of $\mathfrak{H}(X)$

$$
\mathfrak{H}(\Gamma ; X)=\left\{g \in \mathfrak{H}(X) \mid g^{\delta}=0, \text { for all } \delta \in \widehat{K}_{M} \backslash \Gamma\right\} .
$$

The $p$ th Schwartz space $\mathcal{S}^{p}(X)(1<p \leq 2)$ on $X$ is the class of $\mathcal{C}^{\infty}$ functions $f$ on $X$ with the decay condition: for each $D, E \in \mathcal{U}(\mathfrak{g})$ and $n \in \mathbb{Z}^{+} \cup\{0\}$

$$
\mu_{D, E, n}(f):=\sup _{x \in X}|f(D ; x ; E)|(1+|x|)^{n} \varphi_{0}^{-\frac{2}{p}}(x)<+\infty .
$$

The quantities $\mu_{D, E, n}(f)$ gives a countable family of seminorms on the space $\mathcal{S}^{p}(X)$ and the topology induced by this countable family makes $\mathcal{S}^{p}(X)$ a Fréchet space.

Let us denote $\mathcal{S}^{p}(\check{\delta}, X)=\left\{f \in \mathcal{S}^{p}(X) \mid f=d_{\delta} \chi_{\check{\delta}} * f\right\}$ and $\mathcal{S}^{p \delta}(X)=\left\{f^{\delta} \mid f \in \mathcal{S}^{p}(X)\right\}$. Being a closed subspace of $\mathcal{S}^{p}(X)$ the space $\mathcal{S}^{p}(\check{\delta}, X)$ is also a Fréchet space with the topology induced from $\mathcal{S}^{p}(X)$. The $\operatorname{Hom}\left(V_{\delta}, V_{\delta}\right)$ valued function space $\mathcal{S}^{p^{\delta}}(X)$ is also a Fréchet space with respect to the topology induced by the countable family of seminorms: for $D, E \in \mathcal{U}(\mathfrak{g})$ and $n \in \mathbb{Z}^{+} \cup\{0\}$

$$
\mu_{D, E, n}\left(f^{\delta}\right):=\sup _{x \in X}\left\|f^{\delta}(D ; x ; E)\right\|_{\mathbf{2}}(1+|x|)^{n} \varphi_{0}^{-\frac{2}{p}}(x)<+\infty .
$$

Note that for the sake of simplicity we keep the same notation for the seminorms for both the spaces $\mathcal{S}^{p}(X)$ and $\mathcal{S}^{p}(X)$. Clearly the spaces $\mathcal{D}(\check{\delta}, X)$ and $\mathcal{D}^{\delta}(X)$ respectively are dense subspaces of the Schwartz spaces $\mathcal{S}^{p}(\check{\delta}, X)$ and $\mathcal{S}^{p} \delta(X)$ with the respective Schwartz space topologies.

Remark 2.3. The topological isomorphism described in Therem 2.2 can be extended from the Schwartz space $\mathcal{S}^{p} \delta(X)$ onto $\mathcal{S}^{p}(\check{\delta}, X)$.

For any function $f \in \mathcal{S}^{p}(X)$, the Helgason Fourier transform (HFT) $\mathcal{F} f$ is defined by

$$
\mathcal{F} f(\lambda, k M)=\int_{X} f(x) e^{(i \lambda-1) H\left(x^{-1} k\right)} d x .
$$

For $f \in \mathcal{S}^{p}(X)$ the function $\mathcal{F} f$ is defined on the domain $\mathfrak{a}_{\varepsilon}^{*} \times K / M$. Now let $\mathcal{S}\left(\mathfrak{a}_{\varepsilon}^{*} \times K / M\right)$ denote the space consisting of the $\complement^{\infty}$ functions $h$ on $\mathfrak{a}^{*} \times K / M$ which satisfy the following conditions:

(i) For each fixed $k \in K$ the function $\lambda \mapsto h(\lambda, k M)$ extends to Inta $\mathfrak{a}_{\varepsilon}^{*}$ as a holomorphic function and it extends as a continuous function to the closed strip $\mathfrak{a}_{\varepsilon}^{*}$.

(ii) For any $\lambda \in \operatorname{Inta}_{\varepsilon}^{*}$ and $x \in G$ we have $\breve{h}(-\lambda, x)=\check{h}(\lambda, x)$ where

$$
\check{h}(\lambda, x)=\int_{K} h(\lambda, K(x k) M) e^{(i \lambda-1) H(x k)} d k,
$$

where $\mathrm{K}(x k)$ is the $K$ part of $x k \in G$ in the Iwasawa $K A N$ decomposition.

(iii) For each $m, n \in \mathbb{Z}^{+}$and $P \in \mathbf{S}(\mathfrak{a})$

$$
\sup _{\lambda \in \text { Int } \mathfrak{a}_{\mathfrak{\varepsilon}}^{*} ; k \in K / M}\left|P\left(\frac{d}{d \lambda}\right) h\left(\lambda, k: \omega_{\mathfrak{k}}^{m}\right)\right|(1+|\lambda|)^{n}<+\infty
$$

where $\omega_{\mathfrak{k}}$ is the Casimir element of $\mathfrak{k}$.

The space $\mathcal{S}\left(\mathfrak{a}_{\varepsilon}^{*} \times K / M\right)$ becomes a Fréchet space with the topology induced by the countable family of seminorms (2.20). It can be shown that the HFT is a continuous mapping of $\mathcal{S}^{p}(X)$ into $\mathcal{S}\left(\mathfrak{a}_{\varepsilon}^{*} \times K / M\right)$ [EK76, Theorem 3.1]. Let us fix the notation $\mathcal{F} f(\lambda, k M)=\mathcal{F} f(\lambda, k)$. The Inversion formula for HFT for $f \in \mathcal{S}^{p}(X)$ is given by 


$$
f(x)=\frac{1}{2} \int_{\mathfrak{a}^{*}} \int_{K} \mathcal{F} f(\lambda, k) e^{-(i \lambda+1) H\left(x^{-1} k\right)}|\mathbf{c}(\lambda)|^{-2} d \lambda d k .
$$

Here $\mathbf{c}(\lambda)$ is the Harish-Chandra $\mathbf{c}$-function which is completely known (see, [Hel00, Chap. IV]; [GV88, Sect. 4.7]). For our purpose we shall only need the following simple estimate [Ank92] : for constants $c, b>0$

$$
|\mathbf{c}(\lambda)|^{-2} \leq c(|\lambda|+1)^{b} \text { for all } \lambda \in \mathfrak{a}^{*} .
$$

Let $f \in \mathcal{S}^{p}(X)$ then $f^{\delta} \in \mathcal{S}_{\delta}^{p}(X)$. The HFT of $f^{\delta}$ is defined similarly as (2.19) by

$$
\mathcal{F}\left(f^{\delta}\right)(\lambda, k M)=\int_{G} f^{\delta}(x) e^{(i \lambda-1) H\left(x^{-1} k\right)} d x,
$$

here the integration is done on each matrix entry. It can be shown that

$$
\mathcal{F}\left(f^{\delta}\right)(\lambda, k M)=\delta(k) \mathcal{F}\left(f^{\delta}\right)(\lambda, e M)=\delta(k) \widetilde{f^{\delta}}(\lambda),
$$

here we are denoting $\mathcal{F}\left(f^{\delta}\right)(\lambda, e M)$ by $\widetilde{f^{\delta}}(\lambda)$. The function $\widetilde{f^{\delta}}(\lambda)$ can be represented by the following (for details see [JS07]):

$$
\widetilde{f^{\delta}}(\lambda)=d_{\delta} \int_{X} \operatorname{trf} \delta(x) \Phi_{\bar{\lambda}, \delta}^{*}(x) d x
$$

where for each $\delta \in \widehat{K}_{M}$ and $\lambda \in \mathbb{C}$, the function

$$
\Phi_{\lambda, \delta}(x)=\int_{K} e^{-(i \lambda+1) H\left(x^{-1} k\right)} \delta(k) d k, \quad x \in G,
$$

is called the 'generalized spherical function' of class $\delta$. For each $x \in G, \Phi_{\lambda, \delta}(x)$ is an operator in $\operatorname{Hom}\left(V_{\delta}, V_{\delta}\right)$. Taking point-wise adjoints leads to the expression

$$
\Phi_{\bar{\lambda}, \delta}^{*}(x):=\Phi_{\bar{\lambda}, \delta}(x)^{*}=\int_{K} e^{(i \lambda-1) H\left(x^{-1} k\right)} \delta\left(k^{-1}\right) d k, \quad x \in G .
$$

We note that from the Iwasawa decomposition, if $x \in G$ and $\tau \in K, H(\tau x)=H(x)$. Hence, the expressions (2.25) and (2.26) show that both $\Phi_{\lambda, \delta}$ and $\Phi_{\lambda, \delta}^{*}$ can be considered as functions on the space $X=G / K$. The transform $f^{\delta} \mapsto \widetilde{f^{\delta}}$ given by the integral (2.24) will be referred as the $\delta$-spherical transform. We list out some basic properties of the generalized spherical functions $\Phi_{\lambda, \delta}$ in the following remark

Remark 2.4. $\quad$ (i) The following can easily be checked from the integral representations given in (2.25) and (2.26) of the generalized spherical function and it's adjoint

- for all $k \in K, \Phi_{\lambda, \delta}(k x)=\delta(k) \Phi_{\lambda, \delta}(x)$ and $\Phi_{\bar{\lambda}, \delta}^{*}(k x)=\Phi_{\bar{\lambda}, \delta}^{*}(x) \delta\left(k^{-1}\right)$.

- Let $v \in V_{\delta}$ and $m \in M$ then $\delta(m)\left(\Phi_{\bar{\lambda}, \delta}^{*}(x) v\right)=\Phi_{\bar{\lambda}, \delta}^{*}(x) v$.

This shows that $\Phi_{\bar{\lambda}, \delta}^{*}(\cdot)$ is a $\operatorname{Hom}\left(V_{\delta}, V_{\delta}^{M}\right)$ valued function on $X$. Hence, the $\delta$-spherical transform $\widetilde{f^{\delta}}(\cdot)$ as defined in (2.24) is a $\operatorname{Hom}\left(V_{\delta}, V_{\delta}^{M}\right)$ valued function on $\mathbb{C}$.

(ii) For each $\lambda \in \mathbb{C}$ and $\delta \in \widehat{K}_{M}$, the functions $x \mapsto \Phi_{\lambda, \delta}(x)$ is a joint eigenfunction of the algebra $\mathbf{D}(X)$ of all $G$-invariant differential operators on $X$.

(iii) For each fixed $\delta \in \widehat{K}_{M}$ there exists a polynomial [Hel94, Theorem 5.15, ch-III, §5, p-289 ] $Q_{\delta}(1-i \lambda)$ of the complex variable $i \lambda$ such that: for all $\lambda \in \mathbb{C}$

$$
\begin{aligned}
Q_{\delta}(1-i \lambda) \Phi_{\lambda, \delta}(\cdot) & =Q_{\delta}(1+i \lambda) \Phi_{-\lambda, \delta}(\cdot) \\
{\left[Q_{\delta}(1-i \lambda)\right]^{-1} \Phi_{\bar{\lambda}, \delta}^{*}(\cdot) } & =\left[Q_{\delta}(1+i \lambda)\right]^{-1} \Phi_{-\bar{\lambda}, \delta}^{*}(\cdot)
\end{aligned}
$$

Both sides of the above relations are holomorphic for all $\lambda \in \mathbb{C}$. 
The polynomials $Q_{\delta}$ are called the Kostant's polynomials. For a rank-one group the polynomial $Q_{\delta}(1+i \lambda)$ has the following representation interms of the Gamma functions [Hel94, Theorem 11.2, Ch. III, $§ 11]$

$$
Q_{\delta}(1+i \lambda)=\left(\frac{1}{2}(\alpha+\beta+1+i \lambda)\right)_{\frac{r+s}{2}}\left(\frac{1}{2}(\alpha-\beta+1+i \lambda)\right)_{\frac{r-s}{2}}
$$

where, $(z)_{m}=\frac{\Gamma(z+m)}{\Gamma(z)}$. Two group dependent constants $\alpha, \beta$ are given by $\alpha=\frac{1}{2}\left(m_{\gamma}+m_{2 \gamma}-1\right)$, $\beta=\frac{1}{2}\left(m_{2 \gamma}-1\right)$. The pair of integers $(r, s)$ is the parameterization of the representation $\delta \in \widehat{K}_{M}$. Clearly $Q_{\delta}(1+i \lambda)$ is a polynomial in $i \lambda$ of order $2 r$. Helgason [Hel94, Ch. III, $\S 11$ ] further showed that the polynomial $Q_{\delta}(1+i \lambda)$ has no zero in the interior of the strip $\mathfrak{a}_{\varepsilon}^{*}:=\left\{\lambda \in \mathfrak{a}_{\mathbb{C}}^{*}:|\Im \lambda| \leq \varepsilon\right\}$.

We now define a function space in the Fourier domain which is a prospective candidate for the image of $\mathcal{S}^{p^{\delta}}(X)$ under the $\delta$-spherical transform.

Definition 2.5. We denote $\mathcal{S}_{\delta}\left(\mathfrak{a}_{\varepsilon}^{*}\right)$ for the space of all $\operatorname{Hom}\left(V_{\delta}, V_{\delta}\right)$ valued functions $\psi$ on the complex strip $\mathfrak{a}_{\varepsilon}^{*}$ with the properties:

(i) For each $\lambda \in \mathfrak{a}_{\varepsilon}^{*}, \psi(\lambda)$ maps $V_{\delta}$ to $V_{\delta}^{M}$.

(ii) Each $\psi$ is holomorphic in the interior of the strip $\mathfrak{a}_{\varepsilon}^{*}$ and extends as a continuous function to the closed strip.

(iii) $\psi$ satisfies the identity

$$
Q_{\delta}(1-i \lambda)^{-1} \psi(\lambda)=Q_{\delta}(1+i \lambda)^{-1} \psi(-\lambda), \quad \lambda \in \mathfrak{a}_{\varepsilon}^{*},
$$

where $Q_{\delta}(1+i \lambda)$ is the polynomial (2.29).

(iv) For each $P \in \mathbf{S}(\mathfrak{a})$ and for each integer $t \geq 0$ we have:

$$
\tau_{P, t}(\psi)=\sup _{\lambda \in \text { Int } \mathfrak{a}_{\varepsilon}^{*}}\left\|P\left(\frac{d}{d \lambda}\right) \psi(\lambda)\right\|_{2}(1+|\lambda|)^{t}<+\infty .
$$

We have already mentioned that $\operatorname{dim} V_{\delta}^{M}=1$, so for each $\psi \in \mathcal{S}_{\delta}\left(\mathfrak{a}_{\varepsilon}^{*}\right)$ and $\lambda \in \mathfrak{a}_{\varepsilon}^{*}$, with a convenient choice of basis, $\psi(\lambda)$ is a $d_{\delta} \times d_{\delta}$ matrix with all the rows except the first one being identically zero. It can be shown that the space $\mathcal{S}_{\delta}\left(\mathfrak{a}_{\varepsilon}^{*}\right)$ is a Fréchet space with the topology induced by the countable family of seminorms $\left\{\tau_{P, t}\right\}$. We shall be using the following topological characterization of the image of the Schwartz space $\mathcal{S}^{p} \delta(X)$ under the $\delta$-spherical transform.

Theorem 2.6. For $0<p \leq 2$ and $\varepsilon=(2 / p-1)$ the $\delta$-spherical transform $f \mapsto \tilde{f}$ is a topological isomorphism between the spaces $\mathcal{S}^{p} \delta(X)$ and $\mathcal{S}_{\delta}\left(\mathfrak{a}_{\varepsilon}^{*}\right)$.

This is a part of the result proved by Eguchi and Kawata [EK76]. A proof of this theorem avoiding the complicated asymptotics of the generalized spherical functions can be found in [JS07].

\section{Necessary Conditions}

In this section we start with the $L^{p}$-Schwartz space $\mathcal{S}^{p}(X)$ with $0<p \leq 2$. For $f \in \mathcal{S}^{p}(X)$ we define $P_{\lambda} f(x)=\left(f * \varphi_{\lambda}\right)(x)$, for suitable $\lambda \in \mathbb{C}$. We get an alternative expression for the spectral projection $P_{\lambda} f(\cdot)$ in terms of the Helgason Fourier transform $\mathcal{F} f$ of the function $f \in \mathcal{S}^{p}(X)$. Beginning with $P_{\lambda} f(x)=\int_{G} f(y) \varphi_{\lambda}\left(y^{-1} x\right) d y$, we use the standard symmetric property (2.7) of the elementary spherical functions and the Fubini's theorem to write:

$$
\begin{aligned}
P_{\lambda} f(x) & =\int_{K}\left\{\int_{G} f(y) e^{(i \lambda-1) H\left(y^{-1} k^{-1}\right)} d y\right\} e^{-(i \lambda+1) H\left(x^{-1} k^{-1}\right)} d k \\
& =\int_{K} \mathcal{F} f\left(\lambda, k^{-1}\right) e^{-(i \lambda+1) H\left(x^{-1} k^{-1}\right)} d k \\
& =\int_{K} \mathcal{F} f(\lambda, k) e^{-(i \lambda+1) H\left(x^{-1} k\right)} d k
\end{aligned}
$$

We have already mentioned that for any $f \in \mathcal{S}^{p}(X)$ the Helgason Fourier transform $\mathcal{F} f$ is defined on the domain $\mathfrak{a}_{\varepsilon}^{*} \times K / M$. Hence, (3.1) implies that for each $f \in \mathcal{S}^{p}(X)$ the function $(\lambda, x) \mapsto P_{\lambda} f(x)$ is defined on $\mathfrak{a}_{\varepsilon}^{*} \times X$. We use the notation $\mathfrak{e}_{\lambda, k}(x)$ for $e^{-(i \lambda+1) H\left(x^{-1} k\right)}$, which is the kernel of the 
integral in the definition (3.1). As we have already mentioned that the Iwasawa decomposition $K A N$ is diffeomorphic to $G$, so, for each $k \in K$, the Iwasawa-a-projection $x \mapsto H\left(x^{-1} k\right)$ is a $e^{\infty}$ map on $G$. Thus $\mathfrak{e}_{\lambda, k} \in \mathfrak{C}^{\infty}(X)$ for each $\lambda \in \mathbb{C}$ and $k \in K$. Hence, from (3.1) one can conclude that for each $\lambda \in \mathfrak{a}_{\varepsilon}^{*}$ and for each $f \in \mathcal{S}^{p}(X), P_{\lambda} f \in \mathcal{C}^{\infty}(X)$. Furthermore the kernel $\mathfrak{e}_{\lambda, k}$ is a joint eigenfunction of the algebra $\mathbf{D}(X)$. In particular for the Laplace-Beltrami operator $\mathbf{L}$ the eigenvalue for $\mathfrak{e}_{\lambda, k}$ we have:

$$
\mathbf{L}_{\lambda, k}(x)=-\left(1+\lambda^{2}\right) \mathfrak{e}_{\lambda, k}(x),
$$

for each $\lambda \in \mathbb{C}$ and $k \in K$ [GV88]. According to our notation $\mathfrak{e}_{\lambda, k} \in \mathcal{E}_{\lambda}(X)$ for each $\lambda \in \mathbb{C}$. Note that the integral in the definition (3.1) of $P_{\lambda} f$ is over a compact set. Therefore, it follows easily that for each $\lambda \in \mathfrak{a}_{\varepsilon}^{*}$ and $f \in \mathcal{S}^{p}(X), P_{\lambda} f \in \mathcal{E}_{\lambda}(X)$.

To prove other characteristic properties of the function space $P_{\lambda}\left(\mathcal{S}^{p}(X)\right)\left(\lambda \in \mathfrak{a}_{\varepsilon}^{*}\right)$. We shall mainly use the continuity of the $\delta$-spherical transform [JS07, Lemma 4.2] which is a part of Theorem 2.6.

For each $f \in \mathcal{S}^{p}(X)$ and for each $\lambda \in \mathfrak{a}_{\varepsilon}^{*}, P_{\lambda} f \in \mathfrak{C}^{\infty}(X)$, we define its matrix valued left $\delta$-projection $\left(P_{\lambda} f\right)^{\delta}$ by

$$
\left(P_{\lambda} f\right)^{\delta}(x)=d_{\delta} \int_{K} P_{\lambda} f(k x) \delta\left(k^{-1}\right) d k .
$$

It is clear that for each $\delta \in \widehat{K}_{M},\left(P_{\lambda} f\right)^{\delta} \in \mathcal{E}_{\lambda}^{\delta}(X)$. Now $\left(P_{\lambda} f\right)^{\delta}$ satisfies $\left(P_{\lambda} f\right)^{\delta}(k x)=\delta(k)\left(P_{\lambda} f\right)^{\delta}(x)$ $(k \in K, x \in X)$. Hence $\operatorname{tr}\left(P_{\lambda} f\right)^{\delta}$ is a left $\check{\delta}$-type scalar valued function and hence $\operatorname{tr}\left(P_{\lambda} f\right)^{\delta} \in \mathcal{E}_{\lambda}(\check{\delta}, X)$. The following proposition relates the projection $\left(P_{\lambda} f\right)^{\delta}$ with the generalized spherical function $(2.25)$. This structure will be very useful for estimating the decay of the function $P_{\lambda} f$ for each $f \in \mathcal{S}^{p}(X)$ $(0<p \leq 2)$.

Proposition 3.1. Let $f \in L^{p}(X)$ if $1<p \leq 2$ and $f \in \mathcal{S}^{p}(X)$ if $0<p \leq 1$. Then $\left(P_{\lambda} f\right)^{\delta}(x)=$ $P_{\lambda}\left(f^{\delta}\right)(x)=\Phi_{\lambda, \delta}(x) \widetilde{f^{\delta}}(\lambda)$, for $x \in X$ and $\lambda \in$ Int $\mathfrak{a}_{\varepsilon}^{*}$, where $\widetilde{f^{\delta}}$ is the $\delta$-spherical transform of $f^{\delta}$ as defined in (2.13).

Proof. The existence of $P_{\lambda} f$ needs a proof in case of $f \in L^{p}(X), 1<p \leq 2$. It is a consequence of the estimates (2.8) and (2.10) of the function $\varphi_{\lambda}$, that for $\lambda \in \mathfrak{a}_{\varepsilon}^{*}, \varphi_{\lambda} \in L^{q}(X)$, where $\frac{1}{p}+\frac{1}{q}=1$. Further, it can be shown that for each compact set $U \subset$ Inta $\mathfrak{a}_{\varepsilon}^{*}$, there exists a $g \in L^{q}(X)$ with $g \geq 0$ such that $\left|\varphi_{\lambda}(x)\right|<g(x)$, for $\lambda \in U$ and $x \in X$. Thus by Hölder's inequality $f * \varphi_{\lambda}(x)$ exists for all $\lambda \in U$. Moreover, the uniform domination of the $\varphi_{\lambda}$ means that $\lambda \mapsto \varphi_{\lambda}$ is a continuous map of $U$ to $L^{q}(X)$. Hölder's inequality will then make $f * \varphi_{\lambda}(x)$ continuous in $\lambda \in U$. The compact set $U$ being arbitrary we get the existence and continuity in both the variables on $\operatorname{Int} \mathfrak{a}_{\varepsilon}^{*} \times X$. From (3.3) we now have:

$$
\begin{aligned}
\left(P_{\lambda} f\right)^{\delta}(x) & =d_{\delta} \int_{K} P_{\lambda} f(k x) \delta\left(k^{-1}\right) d k \\
& =d_{\delta} \int_{K} \int_{G} f(y) \varphi_{\lambda}\left(y^{-1} k x\right) d y \delta\left(k^{-1}\right) d k \\
& =d_{\delta} \int_{K} \int_{G} f(k z) \varphi_{\lambda}\left(z^{-1} x\right) d z \delta\left(k^{-1}\right) d k \\
& =d_{\delta} \int_{G} \varphi_{\lambda}\left(z^{-1} x\right) \int_{K} f(k z) \delta\left(k^{-1}\right) d k d z \\
& =\int_{G} f^{\delta}(z) \varphi_{\lambda}\left(z^{-1} x\right) d z \\
& =P_{\lambda}\left(f^{\delta}\right)(x) .
\end{aligned}
$$

Using the symmetric property of the elementary spherical function (2.7) the expression (3.4) can be written as

$$
\left(P_{\lambda} f\right)^{\delta}(x)=\int_{G} f^{\delta}(z) \int_{K} e^{-(i \lambda+1) H\left(x^{-1} k\right)} e^{(i \lambda-1) H\left(z^{-1} k\right)} d k d z
$$


The repeated integral on the right-hand side converging absolutely, we interchange the integrals to obtain

$$
\begin{aligned}
\left(P_{\lambda} f\right)^{\delta}(x) & =\int_{K} e^{-(i \lambda+1) H\left(x^{-1} k\right)} \int_{G} f^{\delta}(z) e^{(i \lambda-1) H\left(z^{-1} k\right)} d z d k \\
& =\int_{K} e^{-(i \lambda+1) H\left(x^{-1} k\right)} \mathcal{F} f^{\delta}(\lambda, k) d k
\end{aligned}
$$

We have already noticed that $\mathcal{F} f^{\delta}(\lambda, k)=\delta(k) \mathcal{F} f^{\delta}(\lambda, e)$ and also we have observed that $\mathcal{F} f^{\delta}(\lambda, e)=$ $\widetilde{f^{\delta}}(\lambda)$. Then

$$
\left(P_{\lambda} f\right)^{\delta}(x)=\left\{\int_{K} e^{-(i \lambda+1) H\left(x^{-1} k\right)} \delta(k) d k\right\} \widetilde{f^{\delta}}(\lambda)=\Phi_{\lambda, \delta}(x) \widetilde{f^{\delta}}(\lambda)
$$

Remark 3.2. As $\varphi_{\lambda}(x)=\varphi_{-\lambda}(x)$ for all $\lambda \in \mathbb{C}$, so both the functions $P_{\lambda} f,\left(P_{\lambda} f\right)^{\delta}$ and $\operatorname{tr}\left(P_{\lambda} f\right)^{\delta}$ are even in the $\lambda$ variable.

To characterize $P_{\lambda} f$ for $f \in \mathcal{S}^{p}(X)$, we shall first concentrate on each of its $\delta$-projections $\left(P_{\lambda} f\right)^{\delta}$. The following proposition summarizes the properties of $\left(P_{\lambda} f\right)^{\delta}$ which will be useful to characterize $P_{\lambda}\left(\mathcal{S}^{p}(X)\right)$ $\left(\lambda \in \mathfrak{a}_{\varepsilon}^{*}\right)$.

Proposition 3.3. For $f \in \mathcal{S}^{p}(X)$, where $0<p \leq 2$ and for each fixed $\delta \in \widehat{K}_{M}$ the operator valued left $\delta$-projection $\left(P_{\lambda} f\right)^{\delta}$ of $P_{\lambda} f\left(\lambda \in \mathfrak{a}_{\varepsilon}^{*}\right)$ has the properties:

(i) For each $\lambda \in \mathfrak{a}_{\varepsilon}^{*}$, the function $\left(P_{\lambda} f\right)^{\delta} \in \mathcal{E}_{\lambda}^{\delta}(X)$.

(ii) For each $x \in X, \lambda \mapsto\left(P_{\lambda} f\right)^{\delta}(x)$ is an even holomorphic function in the interior of the strip $\mathfrak{a}_{\varepsilon}^{*}$ and it extends as an even continuous function on the closed strip. The map $\lambda \mapsto Q_{\delta}(1-$ $i \lambda)^{-1}\left(P_{\lambda} f\right)^{\delta}(x)$ is a holomorphic function on the open strip Inta $\mathfrak{a}_{\varepsilon}^{*}$. For $p=2,\left(P_{\lambda} f\right)^{\delta}(x)$ is a real analytic function of $\lambda \in \mathbb{R}$.

(iii) For each $\boldsymbol{D}, \boldsymbol{E} \in \mathcal{U}(\mathfrak{g}), m, n, s \in \mathbb{Z}^{+} \cup\{0\}$ and for any real number $r_{p}<\frac{2 p-2}{p}$ we can find positive constants $c_{i}$ and positive integers $l, t$ such that

$$
\begin{aligned}
& \sup _{x \in G, \lambda \in \text { Int } \mathfrak{a}_{\varepsilon}^{*}} \|\left(\frac{d}{d \lambda}\right)^{m}\left(P_{\lambda} f^{\delta}\right)(\boldsymbol{D} ; x ; \boldsymbol{E}) \|_{\mathbf{2}}(1+|x|)^{n}(1+|\lambda|)^{s} \varphi_{0}^{-r_{p}}(x) \\
& \leq \sum_{i=0}^{m} c_{i}(1+|\delta|)^{q} \sup _{x \in X}\left\|\boldsymbol{L}^{l} f^{\delta}(x)\right\|_{2}(1+|x|)^{t} \varphi_{0}^{-\frac{2}{p}}(x) .
\end{aligned}
$$

Proof. Property (i) has already been discussed. The property (ii) is a consequence of the expression (3.6) and from the fact that the function $\lambda \mapsto \widetilde{f^{\delta}}(\lambda)$ satisfies property (ii) and (iii) of Definition 2.5.

(iii) Using the result of the Proposition 3.1 we get

$$
\begin{aligned}
\|\left(\frac{d}{d \lambda}\right)^{m}\left(P_{\lambda} f\right)^{\delta}(\mathbf{D} ; x ; \mathbf{E}) & \left\|_{2}=\right\|\left(\frac{d}{d \lambda}\right)^{m}\left\{\Phi_{\lambda, \delta}(\mathbf{D} ; x ; \mathbf{E}) \widetilde{f^{\delta}}(\lambda)\right\} \|_{2} \\
& \leq \sum_{\ell=0}^{t}\left\|\left(\frac{d}{d \lambda}\right)^{\ell} \Phi_{\lambda, \delta}(\mathbf{D} ; x ; \mathbf{E})\right\|_{\mathbf{2}}\left\|\left(\frac{d}{d \lambda}\right)^{m-\ell} \widetilde{f^{\delta}}(\lambda)\right\|_{2}
\end{aligned}
$$

We shall use the following estimates for the various derivatives of the matrix coefficients of the principal series representation $[\mathrm{HC} 76, \S 17$, Lemma 1]:

$$
\left\|\left(\frac{d}{d \lambda}\right)^{\ell} \Phi_{\lambda, \delta}(\mathbf{D} ; x ; \mathbf{E})\right\|_{\mathbf{2}} \leq c(1+|\delta|)^{q}(1+|\lambda|)^{q}(1+|x|)^{u} \varphi_{0}(x) e^{|\Im \lambda||x|}
$$

where $c>0$ is a constant (may depend on the derivatives chosen but independent of $\delta \in \widehat{K}_{M}$ ), $q \in \mathbb{Z}^{+}$ depends on $\mathbf{D}, \mathbf{E} \in \mathcal{U}(\mathfrak{g})$ and $u \in \mathbb{Z}^{+}$depends on the integer $\ell$. As $\lambda \in \mathfrak{a}_{\varepsilon}^{*}$ we can replace $|\Im \lambda|$ by 
$\varepsilon=\left(\frac{2}{p}-1\right)$ in (3.9). Now from (3.8) and (3.9) we get:

$$
\begin{aligned}
& \left\|\left(\frac{d}{d \lambda}\right)^{m}\left(P_{\lambda} f\right)^{\delta}(\mathbf{D} ; x ; \mathbf{E})\right\|_{\mathbf{2}}(1+|x|)^{n}(1+|\lambda|)^{s} \varphi_{0}^{-r_{p}}(x) \\
& \quad \leq \sum_{\ell=0}^{m} c_{\ell}(1+|x|)^{n+u}(1+|\lambda|)^{s+q}(1+|\delta|)^{q} \varphi_{0}^{1-r_{p}}(x) e^{\varepsilon|x|}\left\|\left(\frac{d}{d \lambda}\right)^{m-\ell} \widetilde{f^{\delta}}(\lambda)\right\|_{2} .
\end{aligned}
$$

We notice that for all $0<p \leq 2,1-r_{p}>0$. Now we make use of the estimate $\varphi_{0}^{1-r_{p}}(x) \leq a(1+$ $|x|)^{b_{p}} e^{\left(r_{p}-1\right)|x|}, x \in X$ (where $b_{p}$ is a positive real number, to be precise, it is exactly $\left.1-r_{p}\right)$. This is an easy consequence of the two-sided estimate (2.11) of the elementary spherical function $\varphi_{0}(x)$. Hence we can continue the above chain of inequalities by

$$
\leq \sum_{\ell=0}^{m} c_{\ell}(1+|x|)^{n+u+b_{p}}(1+|\lambda|)^{s+q}(1+|\delta|)^{q} e^{-\gamma|x|}\left\|\left(\frac{d}{d \lambda}\right)^{m-\ell} \widetilde{f^{\delta}}(\lambda)\right\|_{\mathbf{2}},
$$

where $\gamma=2-\frac{2}{p}-r_{p}$. Clearly, $\gamma>0$ as $r_{p}<\frac{2 p-2}{p}$. Hence from (3.10)

$$
\begin{aligned}
\sup _{x \in G, \lambda \in \operatorname{Int\mathfrak {a}_{\varepsilon }^{*}},} & \left\|\left(\frac{d}{d \lambda}\right)^{m}\left(P_{\lambda} f\right)^{\delta}(\mathbf{D} ; x ; \mathbf{E})\right\|_{\mathbf{2}}(1+|x|)^{n}(1+|\lambda|)^{s} \varphi_{0}^{-r_{p}}(x) \\
\leq & \sum_{\ell=0}^{t} c_{\ell}(1+|\delta|)^{q}\left\{\sup _{x \in X}(1+|x|)^{n+u+b_{p}} e^{-\gamma|x|}\right\} \\
& \left\{\sup _{\lambda \in \operatorname{Int} \mathfrak{a}_{\varepsilon}^{*}}(1+|\lambda|)^{s+q}\left\|\left(\frac{d}{d \lambda}\right)^{m-\ell} \widetilde{f^{\delta}}(\lambda)\right\|_{\mathbf{2}}\right\} \\
\leq & \left.\sum_{\ell=0}^{m} \overline{c_{\ell}}(1+|\delta|)^{q}\left\{\sup _{\lambda \in \operatorname{Int} \mathfrak{a}_{\varepsilon}^{*}}(1+|\lambda|)^{s+q}\left\|\left(\frac{d}{d \lambda}\right)^{m-\ell} \widetilde{f^{\delta}}(\lambda)\right\|\right\}_{\mathbf{2}}\right\} .
\end{aligned}
$$

Now the expression within braces is the norm $\tau_{s+q, m-\ell}\left(\widetilde{f^{\delta}}\right)$. Using the continuity of the $\delta$-spherical transform, we write: there exists positive integers $l, t$ such that the last expression (3.11) is dominated by

$$
c(1+|\delta|)^{q} \sup _{x \in G}\left\|\mathbf{L}^{l} f^{\delta}(x)\right\|_{\mathbf{2}}(1+|x|)^{t} \varphi_{0}^{-\frac{2}{p}}(x) .
$$

Remark 3.4. The fact that $\lambda \mapsto Q_{\delta}(1-i \lambda)^{-1}\left(P_{\lambda} f\right)^{\delta}$ (for all $f \in \mathcal{S}^{p}(X)$ ) is holomorphic on Inta $\mathfrak{a}_{\varepsilon}^{*}$ can be given a separate proof by using the structural form (3.6) of $\left(P_{\lambda} f\right)^{\delta}(\cdot)$. It can be shown that (in fact we shall discuss about this in detail in the next section), for each $x=k a_{t} K, \Phi_{\lambda, \delta}\left(k a_{t} K\right)=$ $\delta(k) Q_{\delta}(1+i \lambda) \Phi(\lambda, t)$, where $\Phi(\lambda, t)$ is a scalar valued function on $\mathbb{C} \times \overline{\mathbb{R}^{+}}$such that for each value of $\lambda$ it is a nonzero function in the $t$ variable. Hence, by (3.6), $Q_{\delta}(1+i \lambda)^{-1}\left(P_{\lambda} f\right)^{\delta}$ is holomorphic on Inta $\varepsilon_{\varepsilon}^{*}$. Now $\lambda \mapsto\left(P_{\lambda} f\right)^{\delta}$ being an even function, it is easy to notice that actually, $\left[Q_{\delta}(1-i \lambda) Q_{\delta}(1+i \lambda)\right]^{-1}\left(P_{\lambda} f\right)^{\delta}$ is holomorphic on Inta $\mathfrak{a}_{\varepsilon}^{*}$.

The above proposition helps us to get the decay/growth of $P_{\lambda} f$ when $f \in \mathcal{S}^{p}(X)$ and $\lambda \in \mathfrak{a}_{\varepsilon}^{*}$. The following is the main theorem of this section.

Theorem 3.5. For $f \in \mathcal{S}^{p}(X)(0<p \leq 2)$, the complex valued function $P_{\lambda} f$ defined on $\mathfrak{a}_{\varepsilon}^{*} \times X$ has the properties:

(i) For each $\lambda \in \mathfrak{a}_{\varepsilon}^{*}, P_{\lambda} f \in \mathcal{E}_{\lambda}(X)$;

(ii) For each $x \in X$ the function $\lambda \mapsto P_{\lambda} f(x)$ is an even holomorphic function on Inta $\mathfrak{a}_{\varepsilon}^{*}$ and it extends as an even continuous function to the closed strip $\mathfrak{a}_{\varepsilon}^{*}$. For each $\delta \in \widehat{K}_{M}$, the $\delta$-projection $\left(P_{\lambda} f\right)^{\delta}$ is an identically zero function on $X$ at all the zeros of the Kostant polynomial $Q_{\delta}(1-i \lambda)$ lying in $\operatorname{Int} \mathfrak{a}_{\varepsilon}^{*}$; 
(iii) For each $\boldsymbol{D}, \boldsymbol{E} \in \mathcal{U}(\mathfrak{g}), m, n, s \in \mathbb{Z}^{+} \cup\{0\}$ and for all real number $r_{p}<\frac{2 p-2}{p}$, one can find integers $\ell, t \in \mathbb{Z}^{+}$and a positive constant $c$ depending on $m, n, s$ and $r_{p}$ such that:

$$
\begin{array}{r}
\sup _{x \in G, \lambda \in I n t \mathfrak{a}_{\varepsilon}^{*}}\left|\left(\frac{d}{d \lambda}\right)^{m} P_{\lambda} f(\boldsymbol{D} ; x ; \boldsymbol{E})\right|(1+|x|)^{n}(1+|\lambda|)^{s} \varphi_{0}^{-r_{p}}(x) \\
\leq c \sup _{x \in G}\left|\boldsymbol{L}^{\ell} f(x)\right|(1+|x|)^{t} \varphi_{0}^{-\frac{2}{p}}(x)<+\infty .
\end{array}
$$

Proof. The property (i) has already been discussed. Condition (ii) is an easy consequence of the PeterWeyl decomposition

$$
P_{\lambda} f(x)=\sum_{\delta \in \widehat{K}_{M}} \operatorname{tr}\left(P_{\lambda} f\right)^{\delta}(x), \quad \text { for all } x \in X
$$

and Proposition 3.3. To obtain (iii) we use (3.14) to get:

$$
\begin{aligned}
\left|\left(\frac{d}{d \lambda}\right)^{m} P_{\lambda} f(\mathbf{D} ; x ; \mathbf{E})\right| & (1+|x|)^{n}(1+|\lambda|)^{s} \varphi_{0}^{-r_{p}}(x) \\
& \leq \sum_{\delta \in \widehat{K}_{M}}\left|\left(\frac{d}{d \lambda}\right)^{m} \operatorname{tr}\left(P_{\lambda} f\right)^{\delta}(\mathbf{D} ; x ; \mathbf{E})\right|(1+|x|)^{n}(1+|\lambda|)^{s} \varphi_{0}^{-r_{p}}(x) \\
& =\sum_{\delta \in \widehat{K}_{M}}\left|\operatorname{tr}\left[\left(\frac{d}{d \lambda}\right)^{m}\left(P_{\lambda} f\right)^{\delta}\right](\mathbf{D} ; x ; \mathbf{E})\right|(1+|x|)^{n}(1+|\lambda|)^{s} \varphi_{0}^{-r_{p}}(x) \\
& \leq \sum_{\delta \in \widehat{K}_{M}}\left\|\left(\frac{d}{d \lambda}\right)^{m}\left(P_{\lambda} f\right)^{\delta}(\mathbf{D} ; x ; \mathbf{E})\right\|_{2}(1+|x|)^{n}(1+|\lambda|)^{s} \varphi_{0}^{-r_{p}}(x) .
\end{aligned}
$$

The next inequality follows easily from (3.15) by applying (3.11).

$$
\begin{aligned}
& \sup _{x \in G ; \lambda \in \text { Int } \mathfrak{a}_{\varepsilon}^{*}}\left(\frac{d}{d \lambda}\right)^{m} P_{\lambda} f(\mathbf{D} ; x ; \mathbf{E}) \mid(1+|x|)^{n}(1+|\lambda|)^{s} \varphi_{0}^{-r_{p}}(x) \\
& \leq c \sum_{\delta \in \widehat{K}_{M}} \sum_{j=0}^{m} \sup _{\lambda \in \text { Int } \mathfrak{a}_{\varepsilon}^{*}}\left\{(1+|\lambda|)^{s+q}(1+|\delta|)^{q}\left\|\left(\frac{d}{d \lambda}\right)^{m-j} \widetilde{f^{\delta}}(\lambda)\right\|_{\mathbf{2}}\right\} \\
& \leq \sum_{j=1}^{m} \sum_{\delta \in \widehat{K}_{M}}(1+|\delta|)^{-2}\left\{\sup _{\lambda \in \text { Inta a }}(1+|\lambda|)^{s+q}(1+|\delta|)^{q+2}\left\|\left(\frac{d}{d \lambda}\right)^{m-j} \widetilde{f^{\delta}}(\lambda)\right\|_{\mathbf{2}}\right\} .
\end{aligned}
$$

As $f \in \mathcal{S}^{p}(X)$, so its HFT $\mathcal{F} f \in \mathcal{S}\left(\mathfrak{a}_{\varepsilon}^{*} \times K / M\right)$ [EK76]. We have also noticed that the Fréchet topology on $\mathcal{S}\left(\mathfrak{a}_{\varepsilon}^{*} \times K / M\right)$ is induced by the countable family 2.20 of seminorms. By the theory of smooth functions on the compact group [Sug71, Theorem 4], it follows that the topology of $\mathcal{S}\left(\mathfrak{a}_{\varepsilon}^{*} \times K / M\right)$ can also be obtained from the equivalent family of seminorms, given by

$$
\sup _{\lambda \in I n t \mathfrak{a}_{\varepsilon}^{*} ; \delta \in \widehat{K}_{M}}\left\|P\left(\frac{d}{d \lambda}\right) \widetilde{f^{\delta}}(\lambda)\right\|_{2}(1+|\lambda|)^{\mathrm{n}}(1+|\delta|)^{\mathrm{m}}<+\infty .
$$

Hence, we can state that the expression within braces of each of the summands of (3.16) is dominated by the single finite quantity :

$$
\sup _{\lambda \in I n t \mathfrak{a}_{\varepsilon}^{*} ; \delta \in \widehat{K}_{M}}\left\|\left(\frac{d}{d \lambda}\right)^{m-j} \widetilde{f^{\delta}}(\lambda)\right\|_{\mathbf{2}}(1+|\lambda|)^{s+q}(1+|\delta|)^{q+2} .
$$

This coupled with the summability of $\sum(1+|\delta|)^{-2}$ reduces the inequality (3.16) to:

$$
\begin{aligned}
& \sup _{x \in X, \lambda \in I n t \mathfrak{a}_{\varepsilon}^{*}}\left|\left(\frac{d}{d \lambda}\right)^{m} P_{\lambda} f(\mathbf{D} ; x ; \mathbf{E})\right|(1+|x|)^{n}(1+|\lambda|)^{s} \varphi_{0}^{-r_{p}}(x) \\
& \leq c \sum_{j=0}^{m}\left\{\sup _{\lambda \in I n t \mathfrak{a}_{\varepsilon}^{*} ; \delta \in \widehat{K}_{M}}\left\|\left(\frac{d}{d \lambda}\right)^{m-j} \widetilde{f^{\delta}}(\lambda)\right\|_{\mathbf{2}}(1+|\lambda|)^{s+q}(1+|\delta|)^{q+2}\right\} .
\end{aligned}
$$


Again by the equivalence of the seminorms on $\mathcal{S}\left(\mathfrak{a}_{\varepsilon}^{*} \times K / M\right)$, we can find positive integers $m_{1}, m_{2}, m_{3}$ such that the last expression is

$$
\leq c \sup _{\lambda \in \text { Intas } ; \mathfrak{a}_{\varepsilon}^{*} ; k \in K / M}\left|\left(\frac{d}{d \lambda}\right)^{m_{1}} \mathcal{F} f\left(\lambda, k ; \omega_{\mathfrak{k}}^{m_{2}}\right)\right|(1+|\lambda|)^{m_{3}},
$$

and by the continuity of the HFT on the Schwartz space $\mathcal{S}^{p}(X)[\mathrm{EK} 76]$, we get nonnegative integers $\ell, t \in \mathbb{Z}^{+}$such that the above expression is

$$
\leq c \sup _{x \in G}\left|\mathbf{L}^{\ell} f(x)\right|(1+|x|)^{t} \varphi_{0}^{-\frac{2}{p}}(x) .
$$

This completes the proof of theorem.

Remark 3.6. This part of the characterization does not really use the fact that $G$ is of real rank one. We state the analogue of Theorem 3.5 for any Riemannian symmetric space $X=G / K$ where $G$ a noncompact, connected semisimple Lie group with finite center and not necessarily of real rank one. (We continue to use the notation we have established for the real rank one case, the modifications needed are mostly obvious.)

Let $f \in \mathcal{S}^{p}(X)$ with $0<p \leq 2$, then $P_{\lambda} f$ is a complex valued function on $\mathfrak{a}_{\varepsilon}^{*} \times X$ where $\mathfrak{a}_{\varepsilon}^{*}=$ $\left\{\lambda \in \mathfrak{a}_{\mathbb{C}}^{*} \simeq \mathbb{C}^{n} \mid\|w \lambda(X)\| \leq \varepsilon \rho(X)\right.$ for all $X \in \mathfrak{a}$ and $\left.w \in W\right\}$ and $P_{\lambda} f$ satisfies the following properties:

(i) For each $\lambda \in \mathfrak{a}_{\varepsilon}^{*}, P_{\lambda} f \in \mathcal{E}_{\lambda}(X)=\left\{g \in \mathcal{C}^{\infty}(X) \mid \mathbf{L} g=-\left(\|\rho\|^{2}+\langle\lambda, \lambda\rangle_{1}\right) g\right\}$.

(ii) For each $x \in X, \lambda \mapsto P_{\lambda} f(x)$ is a $W$-invariant holomorphic function on the interior of the complex tube $\mathfrak{a}_{\varepsilon}^{*}$ and it extends as a continuous function to the closed tube.

For each $\delta \in \widehat{K}_{M}$, the $\delta$-projection $\left(P_{\lambda} f\right)^{\delta}$ is a $d_{\delta} \times d_{\delta}$ matrix valued function, where $d_{\delta}=\operatorname{dim} V_{\delta}$. Also, $x \mapsto\left(P_{\lambda} f\right)^{\delta}(x)$ identically vanishes on each hypersurface in Int $\mathfrak{a}_{\varepsilon}^{*}$ on which the polynomial $\operatorname{det} Q_{\delta}(\rho-i \lambda)$ vanishes. (Here $Q_{\delta}(\rho-i \lambda)$ is a $\ell_{\delta} \times \ell_{\delta}\left(\ell_{\delta}=\operatorname{dim} V_{\delta}^{M}\right)$ matrix of constant coefficient polynomials in $(\rho-i \lambda)$, being the higher rank analogue of the Kostant polynomial [Hel94, Ch III, $\S 3]$.

(iii) For each $\boldsymbol{D}, \boldsymbol{E} \in \mathcal{U}(\mathfrak{g}), Q \in \mathbf{S}(\mathfrak{a}), n, s \in \mathbb{Z}^{+} \cup\{0\}$ and for all real number $r_{p}<\frac{2 p-2}{p}$, one can find integers $\ell, t \in \mathbb{Z}^{+}$and a positive constant $c$ depending on $n, s, r_{p}$ and degree of the polynomial $Q$ such that:

$$
\begin{aligned}
& \sup _{x \in G, \lambda \in \operatorname{Int} \mathfrak{a}_{\varepsilon}^{*}}\left|Q(\partial \lambda) P_{\lambda} f(\boldsymbol{D} ; x ; \boldsymbol{E})\right|(1+|x|)^{n}(1+\|\lambda\|)^{s} \varphi_{0}^{-r_{p}}(x) \\
& \leq c \sup _{x \in G}\left|\boldsymbol{L}^{\ell} f(x)\right|(1+|x|)^{t} \varphi_{0}^{-\frac{2}{p}}(x)<+\infty,
\end{aligned}
$$

where $Q(\partial \lambda):=Q\left(\frac{\partial}{\partial \lambda_{1}}, \cdots, \frac{\partial}{\partial \lambda_{n}}\right)$.

For each $\varepsilon>0$, let us now define a function space $\mathcal{P}_{\varepsilon}(X)$.

Definition 3.7. For $\varepsilon>0$, then $\mathcal{P}_{\varepsilon}(X)$ denotes the class of functions $(\lambda, x) \mapsto f_{\lambda}(x)$ defined on $\mathfrak{a}_{\varepsilon}^{*} \times X$ and satisfying the following conditions:

(i) For each $x \in X$ the function $\lambda \mapsto f_{\lambda}(x)$ is an even $\mathcal{C}^{\infty}$ function on $\mathfrak{a}^{*}$ and is analytic on the interior of the strip $\mathfrak{a}_{\varepsilon}^{*}=\{\lambda|| \Im \lambda \mid \leq \varepsilon\}$. On the boundary it extends as a continuous function.

(ii) For each $\lambda \in \mathfrak{a}_{\varepsilon}^{*}$ the map $x \mapsto f_{\lambda}(x)$ is a $\mathfrak{C}^{\infty}$ function on $X$, an eigenfunction of $\boldsymbol{L}, f_{\lambda} \in \mathcal{E}_{\lambda}(X)$. Moreover, for each $\delta \in \widehat{K}_{M}$ and $x \in G$, the function $\lambda \mapsto Q_{\delta}(1-i \lambda)^{-1} f_{\lambda}^{\delta}(x)$ is holomorphic on Int $\mathfrak{a}_{\varepsilon}^{*}$.

(iii) For each $\boldsymbol{D}, \boldsymbol{E} \in \mathcal{U}\left(\mathfrak{g}_{\mathbb{C}}\right)$ and $m, n, s \in \mathbb{Z}^{+} \cup\{0\}$

$$
\sup _{x \in G, \lambda \in \operatorname{Int\mathfrak {a}_{\varepsilon }^{*}} \mid}\left|\left(\frac{d}{d \lambda}\right)^{m} f_{\lambda}(\boldsymbol{D} ; x ; \boldsymbol{E})\right|(1+|x|)^{n}(1+|\lambda|)^{s} \varphi_{0}^{-r_{\varepsilon}}(x)<+\infty,
$$

where $r_{\varepsilon}<\frac{1-\varepsilon}{1+\varepsilon}$. 
It is easy to verify that $\mathcal{P}_{\varepsilon}(X)$ is a Fréchet space with the topology induced by the countable family of seminorms (3.19).

We shall conclude this section by restating the Theorem 3.5 in the light of Definition 3.7.

Theorem 3.8. The spectral projection $f \mapsto P_{\lambda} f$ is a continuous map from the Schwartz space $\mathcal{S}^{p}(X)$ $(0<p \leq 2)$ into $\mathcal{P}_{\varepsilon}(X)$, where $\varepsilon=\left(\frac{2}{p}-1\right)$.

In the next section we shall obtain sufficient conditions for the image of $\mathcal{S}^{p}(X)$ under the transform $f \mapsto P_{\lambda} f$. The fact that $G$ is of real rank-one plays a crucial role there.

\section{Sufficient Conditions}

We begin this section with the definition of a specific subspace of the function space $\mathcal{P}_{\varepsilon}(X)$ for each $\varepsilon \geq 0$.

Definition 4.1. We denote by $\mathcal{P}_{\varepsilon}(X)_{K}$, for each $\varepsilon>0$, the class of functions $f_{\lambda}(x)$ in $\mathcal{P}_{\varepsilon}(X)$ which are of left-K-finite type in the $x$ variable, where the finite set of $\delta \in \widehat{K}_{M}$ involved can be chosen independently of $\lambda$.

In this section we shall try to establish a sufficient condition for a measurable function $(\lambda, x) \mapsto$ $f_{\lambda}(x) \in \mathcal{P}_{\varepsilon}(X)_{K}$ to be of the form $P_{\lambda} f(x)$ with some $f \in \mathcal{S}^{p}(X)$ for suitable $0<p \leq 2$. Let us fix one $\varepsilon \geq 0$ and a function $f_{\lambda}(x) \in \mathcal{P}_{\varepsilon}(X)_{K}$.

Because of the decay (3.19) the integral

$$
f_{n}(x):=(-1)^{n} \int_{\mathfrak{a}^{*+}}\left(1+\lambda^{2}\right)^{n} f_{\lambda}(x)|\mathbf{c}(\lambda)|^{-2} d \lambda, \quad\left(n \in \mathbb{Z}^{+}\right)
$$

converges absolutely, where $\mathbf{c}(\lambda)$ is the Harish-Chandra c-function. Let us set $f_{0}=f$, i.e

$$
f(x)=\int_{\mathfrak{a}^{*+}} f_{\lambda}(x)|\mathbf{c}(\lambda)|^{-2} d \lambda .
$$

It also follows from the specified decay (3.19) of the function $(\lambda, x) \mapsto f_{\lambda}(x)$ : that for $n=0,1, \cdots$, the function $f_{n} \in \mathrm{e}^{\infty}(X)$. As $f_{\lambda}(\cdot) \in \mathcal{E}_{\lambda}(X)$, it can be shown that $\mathbf{L}^{n} f=f_{n}$.

For each $\lambda \in \mathfrak{a}_{\varepsilon}^{*}$ and $\delta \in \widehat{K}_{M}$ we define the operator valued left $\delta$-projection by

$$
f_{\lambda}^{\delta}(x)=\int_{K} f_{\lambda}(k x) \delta\left(k^{-1}\right) d k .
$$

It is clear from the definition of the function space $\mathcal{P}_{\varepsilon}(X)_{K}$ that for each $\delta \in \widehat{K}_{M}$ and each $\lambda \in \mathfrak{a}_{\varepsilon}^{*}$, $f_{\lambda}^{\delta} \in \mathcal{E}_{\lambda}^{\delta}(X)$. The Peter-Weyl decomposition of the function $f_{\lambda}(\cdot)$ is as follows

$$
f_{\lambda}(x)=\sum_{\delta \in \widehat{K}_{M}} \operatorname{tr} f_{\lambda}^{\delta}(x), \lambda \in \mathfrak{a}_{\varepsilon}^{*} .
$$

As $f_{\lambda}$ is assumed to be left $K$-finite, so in the above decomposition (4.4) all but finitely many terms are identically zero functions. Let us denote $\Gamma(f)$ for the finite subset of $\widehat{K}_{M}$ corresponding to the function $f_{\lambda}$ for which the summands are non zero functions. It follows from the earlier discussion that for each $\delta \in \Gamma(f)$ and $\lambda \in \mathfrak{a}_{\varepsilon}^{*}, x \mapsto \operatorname{tr} f_{\lambda}^{\delta}(x)$ is of left $\check{\delta}$-type. Hence $\operatorname{tr} f_{\lambda}^{\delta}(\cdot) \in \mathcal{E}_{\lambda}(\check{\delta}, X)$.

Lemma 4.2. For each $\delta \in \Gamma(f)$ the map $(\lambda, x) \mapsto f_{\lambda}^{\delta}(x)$ satisfies the decay

$$
\sup _{x \in X ; \lambda \in \operatorname{Inta}_{\varepsilon}^{*}}\left\|\left(\frac{d}{d \lambda}\right)^{m} f_{\lambda}^{\delta}(x)\right\|_{2}(1+|x|)^{n}(1+|\lambda|)^{s} \varphi_{0}^{-r_{\varepsilon}}(x)<K<+\infty
$$

where $K=d_{\delta}^{3 / 2} \cdot c$, the constant $c$ being independent of $\delta$. 
Proof. The assertion is true because

$$
\begin{aligned}
& \left\{\left(\frac{d}{d \lambda}\right)^{m} f_{\lambda}^{\delta}(x)\right\}(1+|x|)^{n}(1+|\lambda|)^{s} \varphi_{0}^{-r_{\varepsilon}}(x) \\
& =d_{\delta}\left\{\int_{K}\left(\frac{d}{d \lambda}\right)^{m} f_{\lambda}(k x) \delta\left(k^{-1}\right) d k\right\}(1+|x|)^{n}(1+|\lambda|)^{s} \varphi_{0}^{-r_{\varepsilon}}(x) \\
& =d_{\delta} \int_{K}\left\{\left(\frac{d}{d \lambda}\right)^{m} f_{\lambda}(k x)\right\} \delta\left(k^{-1}\right)(1+|k x|)^{n}(1+|\lambda|)^{s} \varphi_{0}^{-r_{\varepsilon}}(k x) d k
\end{aligned}
$$

Now taking Hilbert Schmidt norms on both sides and using the fact $\|\delta(k)\|_{\mathbf{2}}=\sqrt{d_{\delta}}$ we get an inequality from which we get the required conclusion. ( It is easy to check that the $\delta$ dependent part in the dominating constant is precisely $\left.d_{\delta}^{\frac{3}{2}}\right)$.

An immediate corollary is the following.

Corollary 4.3. For each $m, n, s \in \mathbb{Z}^{+} \cup\{0\}$ and for each fixed $\delta \in \Gamma(f)$

$$
\sup _{x \in X ; \lambda \in I n t \mathfrak{a}_{\varepsilon}^{*}}\left|\left(\frac{d}{d \lambda}\right)^{m} \operatorname{tr} f_{\lambda}^{\delta}(x)\right|(1+|x|)^{n}(1+|\lambda|)^{s} \varphi_{0}^{-r_{\varepsilon}}(x)<+\infty .
$$

Lemma 4.4. The function $f$ obtained in (4.2) is also left-K-finite and moreover,

$$
\operatorname{tr} f^{\delta}(x)=\int_{\mathfrak{a}^{*+}} \operatorname{tr} f_{\lambda}^{\delta}(x)|\boldsymbol{c}(\lambda)|^{-2} d \lambda
$$

Proof. Using (4.2) and (4.4) we get the following:

$$
\begin{aligned}
f(x) & =\int_{\mathfrak{a}^{*+}} \sum_{\delta \in \Gamma(f)} \operatorname{tr} f_{\lambda}^{\delta}(x)|\mathbf{c}(\lambda)|^{-2} d \lambda, \\
& =\sum_{\delta \in \Gamma(f)} \int_{\mathfrak{a}^{*+}} \operatorname{tr} f_{\lambda}^{\delta}(x)|\mathbf{c}(\lambda)|^{-2} d \lambda .
\end{aligned}
$$

Let us denote $\psi_{\delta}(x)=\int_{\mathfrak{a}^{*+}} \operatorname{tr} f_{\lambda}^{\delta}(x)|\mathbf{c}(\lambda)|^{-2} d \lambda$. The integral converges absolutely because of the decay (4.6). We have already noticed that $\operatorname{tr} f_{\lambda}^{\delta}(\cdot)$ are of left $\check{\delta}$-type and we only need the routine checking

$$
\begin{aligned}
\psi_{\delta}(x) & =d_{\delta} \int_{\mathfrak{a}^{*+}}\left\{\chi_{\check{\delta}} * \operatorname{tr} f_{\lambda}^{\delta}\right\}(x)|\mathbf{c}(\lambda)|^{-2} d \lambda \\
& =d_{\delta} \int_{\mathfrak{a}^{*+}}\left\{\int_{K} \chi_{\check{\delta}}\left(k^{-1}\right) \operatorname{tr} f_{\lambda}^{\delta}(k x) d k\right\}|\mathbf{c}(\lambda)|^{-2} d \lambda \\
& =d_{\delta} \int_{K} \chi_{\check{\delta}}\left(k^{-1}\right)\left\{\int_{\mathfrak{a}^{*+}} \operatorname{tr} f_{\lambda}^{\delta}(k x) d k|\mathbf{c}(\lambda)|^{-2} d \lambda\right\} d k \\
& =d_{\delta}\left\{\chi_{\check{\delta}} * \psi_{\delta}\right\}(x)
\end{aligned}
$$

to conclude that each $\psi_{\delta}$ is a scalar valued left $\check{\delta}$-type. The rest follows from the Peter-Weyl decomposition.

So far we have noted that the function $f$ obtained in $(4.2)$ is in $\complement^{\infty}(X)$ and it is of left- $K$-finite type. Now we shall try to show that $f \in \mathcal{S}^{p}(X)$ for some $0<p \leq 2$. Towards that we shall first try to obtain a structural form of $f_{\lambda}^{\delta}$ analogous to the one given in Proposition 3.1. The assumption that $f_{\lambda}^{\delta} \in \mathcal{E}_{\lambda}^{\delta}(X)$ will now play a crucial role. The following theorem is the key to the desired form of $f_{\lambda}^{\delta}$. Let $\delta \in \widehat{K}_{M}$ and $V_{\delta}\left(d_{\delta}=\operatorname{dim} V_{\delta}\right)$ be the representation space for $\delta$ with the orthonormal basis $v_{1}, v_{2}, \cdots, v_{d_{\delta}}$ where $V_{\delta}^{M}=\mathbb{C} v_{1}$.

Theorem 4.5. [Helgason, [Hel70, Theorem 1.4, p-133]]

Let $\lambda \in \mathbb{C}$ be such that $\Re\langle i \lambda, \alpha\rangle \geq 0$, where $\alpha$ is positive restricted root. Then the functions

$$
\Psi_{\lambda, \check{\delta} j}(x)=\sqrt{d_{\delta}} \int_{K} e^{-(i \lambda+1) H\left(x^{-1} k\right)}\left\langle\delta(k) v_{1}, v_{j}\right\rangle d k, \quad 1 \leq j \leq d_{\delta},
$$

form a basis of the eigenspace $\mathcal{E}_{\lambda}(\check{\delta}, X)$. 
We note that by using the definition (2.25) of the generalized spherical functions we can write the basis vectors as follows

$$
\Psi_{\lambda, \check{\delta} j}(x)=\sqrt{d_{\delta}}\left\langle\Phi_{\lambda, \delta}(x) v_{1}, v_{j}\right\rangle .
$$

Remark 4.6. For a group $G$ with real rank one, we have identified the Iwasawa-A-subgroup with $\mathbb{R}$. With this normalization $\mathfrak{a}, \mathfrak{a}^{*}$ are identified with $\mathbb{R}$ and $\mathfrak{a}^{+}$, $\mathfrak{a}^{*+}$ with $\mathbb{R}^{+}$. As we are only considering the real rank one group, so there will be a smallest positive restricted root $\alpha$ and at most one more which will be $2 \alpha$. Clearly, $\alpha \in \mathbb{R}^{+}$. This immediately suggests that for all $\lambda$ with $\Im \lambda \leq 0, \Re\langle i \lambda, \alpha\rangle \geq 0$.

Hence, for all $\lambda \in\left\{\lambda \in \mathfrak{a}_{\varepsilon}^{*} \mid \Im \lambda \leq 0\right\}$, the vectors $\Psi_{\lambda, \check{\delta} j}(x)$ forms a basis of $\mathcal{E}_{\lambda}(\check{\delta}, X)$.

Lemma 4.7. For each $\delta \in \widehat{K}_{M}$ the matrix valued projection $f_{\lambda}^{\delta}$ of $f_{\lambda}$ for each $\lambda \in \mathfrak{a}_{\varepsilon}^{*}$ has the following structural form

$$
f_{\lambda}^{\delta}\left(k a_{t} .0\right)=\sqrt{d_{\delta}} \Phi_{\lambda, \delta}\left(k a_{t} .0\right) h^{\delta}(\lambda)
$$

where $\Phi_{\lambda, \delta}\left(k a_{t} .0\right)$ is a $\left(d_{\delta} \times 1\right)$ matrix and $h^{\delta}(\lambda)$ is a $\left(1 \times d_{\delta}\right)$ matrix

Proof. We note that by assumption the function $\lambda \mapsto f_{\lambda}^{\delta}(\lambda)$ is even for all $x \in X$. Therefore it is enough to establish the structural form (4.12) for $\lambda$ 's in $\mathfrak{a}_{\varepsilon}^{*}$ with $\Im \lambda \leq 0$.

We have noticed that $\operatorname{tr} f_{\lambda}^{\delta} \in \mathcal{E}_{\lambda}(\check{\delta}, X)$ for all $\lambda \in \mathfrak{a}_{\varepsilon}^{*}$ and $\delta \in \Gamma(f)$. Hence for $\lambda \in\left\{\lambda \in \mathfrak{a}_{\varepsilon}^{*} \mid \Im \lambda \leq 0\right\}$ we write $\operatorname{tr} f_{\lambda}^{\delta}(x)$ in terms of the basis vectors given in (4.11) as follows.

$$
\operatorname{tr} f_{\lambda}^{\delta}(x)=\sqrt{d_{\delta}} \sum_{j=1}^{d_{\delta}} h_{j}^{\delta}(\lambda)\left\langle\Phi_{\lambda, \delta}(x) v_{1}, v_{j}\right\rangle
$$

where $h_{j}^{\delta}(\lambda)$ are coefficients depending on $\lambda$. Let us denote the $\left(1 \times d_{\delta}\right)$ matrix $h^{\delta}(\lambda)=\left(h_{1}^{\delta}(\lambda), \cdots, h_{d_{\delta}}^{\delta}(\lambda)\right)$. We also recall the fact that the generalized spherical function $\Phi_{\lambda, \delta}(x)$ vanishes on the orthogonal complement of $V_{\delta}^{M}$, so we can regard $\Phi_{\lambda, \delta}(x)$ as the $\left(d_{\delta} \times 1\right)$ column vector with the entries $\left\langle\Phi_{\lambda, \delta}(x) v_{1}, v_{j}\right\rangle$. Then it is clear from (4.13) that

$$
\operatorname{tr} f_{\lambda}^{\delta}(x)=\sqrt{d_{\delta}} \operatorname{tr}\left[\Phi_{\lambda, \delta}(x) h^{\delta}(\lambda)\right]
$$

Next we shall show that the matrices $f_{\lambda}^{\delta}(x)$ and $\Phi_{\lambda, \delta}(x) h^{\delta}(\lambda)$ have identical entries.

$$
\begin{aligned}
f_{\lambda}^{\delta}(x)_{\imath \ell} & =\left\langle f_{\lambda}^{\delta}(x) v_{\ell}, v_{\imath}\right\rangle \\
& =d_{\delta}\left\langle\int_{K} \operatorname{tr} f_{\lambda}^{\delta}(k x) \delta\left(k^{-1}\right) d k v_{\ell}, v_{\imath}\right\rangle \\
& =d_{\delta} \int_{K} \operatorname{tr} f_{\lambda}^{\delta}(k x)\left\langle\delta\left(k^{-1}\right) v_{\ell}, v_{\imath}\right\rangle d k .
\end{aligned}
$$

Now we use (4.14) to replace $\operatorname{tr} f_{\lambda}^{\delta}(k x)$ to get

$$
\begin{aligned}
& f_{\lambda}^{\delta}(x)_{\imath \ell}=d_{\delta}^{\frac{3}{2}} \sum_{j=1}^{d_{\delta}} \int_{K}\left\langle\Phi_{\lambda, \delta}(k x) v_{1}, v_{j}\right\rangle\left\langle\delta\left(k^{-1}\right) v_{\ell}, v_{\imath}\right\rangle h_{j}^{\delta}(\lambda) d k \\
& =d_{\delta}^{\frac{3}{2}} \sum_{j=1}^{d_{\delta}} \int_{K}\left\langle\delta(k) \Phi_{\lambda, \delta}(x) v_{1}, v_{j}\right\rangle\left\langle\delta\left(k^{-1}\right) v_{\ell}, v_{\imath}\right\rangle h_{j}^{\delta}(\lambda) d k \\
& =d_{\delta}^{\frac{3}{2}} \sum_{j=1}^{d_{\delta}} \int_{K} \overline{\left\langle\delta\left(k^{-1}\right) v_{j}, \Phi_{\lambda, \delta}(x) v_{1}\right\rangle}\left\langle\delta\left(k^{-1}\right) v_{\ell}, v_{\imath}\right\rangle h_{j}^{\delta}(\lambda) d k .
\end{aligned}
$$

The representation coefficients $k \mapsto\langle\delta(k) v, u\rangle\left(u, v \in V_{\delta}\right)$ satisfy the following consequences of the 'Schur's Orthogonality Relations' : If $u, v, u^{\prime}, v^{\prime} \in V_{\delta}$, then

$$
\int_{K}\langle\delta(k) u, v\rangle \overline{\left\langle\delta(k) u^{\prime}, v^{\prime}\right\rangle}=d_{\delta}^{-1}\left\langle u, u^{\prime}\right\rangle \overline{\left\langle v, v^{\prime}\right\rangle} .
$$

Using (4.17) in (4.16) as also the fact that $\left\{v_{i}\right\}\left(1 \leq i \leq d_{\delta}\right)$ forms an orthonormal basis of the representation space $V_{\delta}$ we write

$$
f_{\lambda}^{\delta}(x)_{\imath \ell}=\sqrt{d_{\delta}}\left\langle\Phi_{\lambda, \delta}(x) v_{1}, v_{\imath}\right\rangle h_{\ell}^{\delta}(\lambda)
$$


The right hand side of (4.18) is precisely the $(\imath, \ell)$ entry of the matrix $\sqrt{d_{\delta}} \Phi_{\lambda, \delta}(x) h^{\delta}(\lambda)$. Hence the Lemma follows.

Remark 4.8.

$$
\text { Write } \quad \begin{aligned}
f_{\lambda}^{\delta}(x) & =\sqrt{d_{\delta}} \Phi_{\lambda, \delta}(x) h^{\delta}(\lambda) \\
& =\sqrt{d_{\delta}}\left\{Q_{\delta}(1-i \lambda) \Phi_{\lambda, \delta}(x)\right\}\left\{Q_{\delta}(1-i \lambda)^{-1} h^{\delta}(\lambda)\right\} .
\end{aligned}
$$

We notice that $f_{\lambda}^{\delta}$ is even in the $\lambda$ variable and the function $\lambda \mapsto Q_{\delta}(1-i \lambda) \Phi_{\lambda, \delta}(x)$ is even by (2.27). Hence for all $\lambda \in \mathfrak{a}_{\varepsilon}^{*}$, the function $\lambda \mapsto Q_{\delta}(1-i \lambda)^{-1} h^{\delta}(\lambda)$ is an even function.

At this point we need to look in a different direction. The matrix entries of the generalized spherical functions are associated with Jacobi functions. Let $x=k a_{t} .0 \in X$. Then

$$
\Phi_{\lambda, \delta}\left(k a_{t}\right)=\left\langle\Phi_{\lambda, \delta}\left(k a_{t}\right) v_{1}, v_{j}\right\rangle=\left\langle\delta(k) \Phi_{\lambda, \delta}\left(a_{t}\right) v_{1}, v_{j}\right\rangle .
$$

It can easily be seen that $\Phi_{\lambda, \delta}\left(a_{t}\right) v \in V_{\delta}^{M}$ for all $v \in V_{\delta}$. Hence on $V_{\delta}^{M}, \Phi_{\lambda, \delta}\left(a_{t}\right)$ will be a multiplication operator

$$
\Phi_{\lambda, \delta}\left(a_{t}\right) v_{1}=\varphi_{\lambda, \delta}(t) v_{1},
$$

where $\varphi_{\lambda, \delta}(t)$ is a function of $t$ depending on $\lambda$ and $\delta$. For each $\delta \in \widehat{K}_{M}$ and $\lambda \in \mathfrak{a}_{\mathbb{C}}^{*}$ the function $t \mapsto \varphi_{\lambda, \delta}(t)$ has an expression in terms of the hypergeometric functions (Helgason [Hel74], Koornwinder [Koo84] )

$$
\varphi_{\lambda, \delta}(t)=Q_{\delta}(i \lambda+1)(\alpha+1)_{r}^{-1}(\sinh t)^{r}(\cosh t)^{s} \varphi_{\lambda}^{\alpha+r, \beta+s}(t),
$$

where $\varphi_{\lambda}^{\alpha+r, \beta+s}$ is the Jacobi function of the first kind with parameters $(\alpha+r, \beta+s)$. The integers $(r, s)$ and the quantities $\alpha, \beta$ are already introduced in (2.29). This Jacobi function has the integral representation [Koo84]:

$$
\varphi_{\lambda}^{\alpha+r, \beta+s}(t)=\int_{0}^{1} \int_{0}^{\pi}\left|\cosh t-\mathfrak{r} e^{i \theta} \sinh t\right|^{-i \lambda-\varrho} d P_{\alpha+r, \beta+s}(\mathfrak{r}, \theta),
$$

where $\varrho=\alpha+r+\beta+s+1$ and $d P_{\alpha+r, \beta+s}(\mathfrak{r}, \theta)$ is a probability measure [Hel87] on $[0,1] \times[0, \pi]$.

Lemma 4.9. For all $\lambda \in \mathbb{C}$ the Jacobi function $\varphi_{\lambda}^{\alpha+r, \beta+s}$ satisfies the following:

$$
\begin{aligned}
\varphi_{\lambda}^{\alpha+r, \beta+s}(0) & =1 \\
\left|\left(\frac{d}{d \lambda}\right)^{k} \varphi_{\lambda}^{\alpha+r, \beta+s}(t)\right| & \leq c t^{k} e^{(|\Im \lambda|+\varrho) t}, t \in \mathbb{R}^{+}, k \in \mathbb{Z}^{+} .
\end{aligned}
$$

Proof. These two properties follow from the integral representation (4.22). We use the estimate

$$
\log \left|\cosh t-r e^{i \theta} \sinh t\right| \leq c t
$$

for all $t>0$ to get the inequality (4.24).

Our next Lemma concerns the domain on which the function $h^{\delta}$ is holomorphic.

Lemma 4.10. For each $\delta \in \widehat{K}_{M}$, the functions $\lambda \mapsto h^{\delta}(\lambda)$ and $\lambda \mapsto Q_{\delta}(1-i \lambda)^{-1} h^{\delta}(\lambda)=g^{\delta}(\lambda)$ are holomorphic in the interior of the complex strip $\mathfrak{a}_{\varepsilon}^{*}$.

Proof. We note that the zeros of the polynomial $Q_{\delta}(1-i \lambda)$ are purely imaginary. We have assumed that $x \mapsto f_{\lambda}^{\delta}(x)$ is an identically zero function on $X$ for all $\lambda$ which are zeros of the polynomial $Q_{\delta}(1-i \lambda)$. Also we have assumed that $f_{\lambda}^{\delta}$ is even in $\lambda$. So, $x \mapsto f_{\lambda}^{\delta}(x)$ is also zero for the zeros of $Q_{\delta}(1+i \lambda)$ in Int $\mathfrak{a}_{\varepsilon}^{*}$. Hence, $\lambda \mapsto Q_{\delta}(1+i \lambda)^{-1} f_{\lambda}^{\delta}(\cdot)$ is holomorphic on Int $\mathfrak{a}_{\varepsilon}^{*}$.

We restrict the function $f_{\lambda}^{\delta}(\cdot)$ to $\left(\mathfrak{a}_{\varepsilon}^{*} \times A^{+}\right)$. Then by the structural form obtained in Lemma 4.7 we write:

$$
f_{\lambda}^{\delta}\left(a_{t}\right)=\sqrt{d_{\delta}} \Phi_{\lambda, \delta}\left(a_{t}\right) h^{\delta}(\lambda), \quad(t>0)
$$


For proving $\lambda \mapsto h^{\delta}(\lambda)$ is holomorphic on $\operatorname{Int} \mathfrak{a}_{\varepsilon}^{*}$ it is enough to prove that each of its matrix entries is so. By definition the $(1, j)$ th $\left(1 \leq j \leq d_{\delta}\right)$ matrix entry of $f_{\lambda}^{\delta}\left(a_{t}\right)$ is given by $f_{\lambda}^{\delta}\left(a_{t}\right)_{1 j}=\sqrt{d_{\delta}} \Phi_{\lambda, \delta}{ }_{1}\left(a_{t}\right) h^{\delta}(\lambda)_{j}$.

$$
\Phi_{\lambda, \delta 1}\left(a_{t}\right)=\left\langle\Phi_{\lambda, \delta}\left(a_{t}\right) v_{1}, v_{1}\right\rangle=\left\|v_{1}\right\| \varphi_{\lambda, \delta}(t) .
$$

Hence by (4.26) and the expression (4.21) we get:

$$
f_{\lambda}^{\delta}\left(a_{t}\right)_{1 j}=\left\|v_{1}\right\|(\alpha+1)_{r}^{-1} Q_{\delta}(1+i \lambda)(\sinh t)^{r}(\cosh t)^{s} \varphi_{\lambda}^{\alpha+r, \beta+s}(t) h_{j}^{\delta}(\lambda) .
$$

As the first order zeros of $Q_{\delta}(1+i \lambda)$ are neutralized by that of $f_{\lambda}^{\delta}\left(a_{t}\right)_{1 j}$, so we write:

$$
Q_{\delta}(1+i \lambda)^{-1} f_{\lambda}^{\delta}\left(a_{t}\right)_{1 j}=C_{v_{1}, \alpha}(\sinh t)^{r}(\cosh t)^{s} \varphi_{\lambda}^{\alpha+r, \beta+s}(t) h_{j}^{\delta}(\lambda) .
$$

The left hand side of (4.28) is holomorphic on $\operatorname{Int} \mathfrak{a}_{\varepsilon}^{*}$. To conclude that $h_{j}^{\delta}$ is holomorphic at $\lambda \in \operatorname{Int} \mathfrak{a}_{\varepsilon}^{*}$, we can choose $t_{0}>0$ so that $\varphi_{\lambda}^{\alpha+r, \beta+s}\left(t_{0}\right) \neq 0$ as is possible by the observation (4.23). Noting that $\varphi_{\lambda}^{\alpha+r, \beta+s}\left(t_{0}\right)$ is holomorphic in $\lambda$ and that both $\sinh t_{0}$ and $\cosh t_{0}$ are positive we reach our conclusion. To see that $\lambda \mapsto Q_{\delta}(1-i \lambda)^{-1} h^{\delta}(\lambda)$ is holomorphic on Int $\mathfrak{a}_{\varepsilon}^{*}$, we note that $f_{\lambda}^{\delta}\left(a_{t}\right)_{1 j}$ is symmetric in $\lambda$ and so from (4.28) $Q_{\delta}(1+i \lambda)^{-1} f_{\lambda}^{\delta}\left(a_{t}\right)_{1 j}$ as well as $Q_{\delta}(1-i \lambda)^{-1} f_{\lambda}^{\delta}\left(a_{t}\right)_{1 j}$ are analytic in Inta $\mathfrak{a}_{\varepsilon}^{*}$. From the exact expression (2.29) of $Q_{\delta}(1-i \lambda)$ we further notice that the polynomials $Q_{\delta}(1+i \lambda)$ and $Q_{\delta}(1-i \lambda)$ have no common zeros. We can hence conclude that $\left[Q_{\delta}(1-i \lambda) Q_{\delta}(1+i \lambda)\right]^{-1} f_{\lambda}^{\delta}\left(a_{t}\right)_{1 j}$ is analytic on Int $\mathfrak{a}_{\varepsilon}^{*}$. Using (4.28) again we get the desired analyticity of $Q_{\delta}(1-i \lambda)^{-1} h^{\delta}(\lambda)$ in Inta $\mathfrak{a}_{\varepsilon}^{*}$.

Remark 4.11. For each $x \in X$ and $\delta \in \widehat{K}_{M} ; \lambda \mapsto f_{\lambda}^{\delta}(x)$ extends as a continuous function to the closed strip $\mathfrak{a}_{\varepsilon}^{*}$. From (4.12) it follows that $\lambda \mapsto h^{\delta}(\lambda)$ also extends as a continuous function to $\mathfrak{a}_{\varepsilon}^{*}$.

Our next aim is to determine the decay of the function $h^{\delta}$, for that we need a lower bound of the associated Jacobi function.

Lemma 4.12. (Bray [Bra96, Lemma 2.4])

Let $\mu, \tau \geq-\frac{1}{2}$, then for any $\Lambda>\frac{2}{\pi}$, there is a constant $C$ depending on $\mu, \tau, \Lambda$ such that

$$
\left|\varphi_{\lambda}^{\mu, \tau}\left(\frac{1}{|\lambda|^{2}}\right)\right| \geq C_{\Lambda, \mu, \tau}, \text { for }|\lambda|>\Lambda
$$

In fact the constant $C_{\Lambda, \mu, \tau}$ has the following form:

$$
C_{\Lambda, \mu, \tau}=e^{-\frac{2+\mu+\tau}{\Lambda}} \cos (1 / \Lambda) .
$$

Remark 4.13. We note that for $G=S U(n, 1)$, the quantities $\alpha \geq 0, \beta=0$ (where $\alpha, \beta$ are as in (2.29)) and the parameterization $(r, s)$ of $\widehat{K}_{M}$ runs over $\mathbb{Z}^{+} \times \mathbb{Z}$ with $r \pm s \in 2 \mathbb{Z}^{+}$. Suppose for some $\delta \in \widehat{K}_{M}, s_{\delta}<0$. In such a case we use the relation [Koo84, (5.75)]

$$
\varphi_{\lambda}^{\alpha+r_{\delta}, s_{\delta}}(t)=(\cosh t)^{2\left|s_{\delta}\right|} \varphi_{\lambda}^{\alpha+r_{\delta},\left|s_{\delta}\right|}(t), t>0, \lambda \in \mathbb{C},
$$

and rewrite (4.21) as follows

$$
\varphi_{\lambda, \delta}(t)=Q_{\delta}(i \lambda+1)(\alpha+1)_{r_{\delta}}^{-1}(\sinh t)^{r_{\delta}}(\cosh t)^{\left|s_{\delta}\right|} \varphi_{\lambda}^{\alpha+r_{\delta},\left|s_{\delta}\right|}(t) .
$$

The Jacobi function $\varphi_{\lambda}^{\alpha+r_{\delta},\left|s_{\delta}\right|}(t)$ clearly satisfies the conditions of Lemma 4.12. For other classes of real rank one groups the parameters $\alpha+r$ and $\beta+s$ are positive integers. Thus, for all $G$ of real rank-one, the condition of Lemma 4.12 holds for the function $\varphi_{\lambda}^{\alpha+r, \beta+s}$.

Proposition 4.14. For each $\delta \in \Gamma(f)$ the function $\lambda \mapsto h_{i}^{\delta}(\lambda)$ for each $i=1,2, \cdots, d_{\delta}$ satisfies the following decay condition:

$$
\sup _{\lambda \in \mathfrak{a}_{\varepsilon}^{*}}\left|\left(\frac{d}{d \lambda}\right)^{n} h_{i}^{\delta}(\lambda)\right|(1+|\lambda|)^{m}<+\infty .
$$

Proof. The structural form obtained in Lemma 4.7 and (4.5) gives the following decay/growth condition for each $(1, j)$ th matrix entry of $f_{\lambda}^{\delta}\left(a_{t}\right)$ : for each $m, n \in \mathbb{Z}^{+} \cup\{0\}$

$$
\sup _{a_{t} \in \mathfrak{a}^{+} ; \lambda \in \operatorname{Int\mathfrak {a}_{\varepsilon }^{*}}}\left|\Phi_{\lambda, \delta 1}\left(a_{t}\right) h_{j}^{\delta}(\lambda)\right|(1+t)^{n}(1+|\lambda|)^{m} \varphi_{0}^{-r_{\varepsilon}}\left(a_{t}\right)=c_{1 j}<+\infty .
$$


This immediately implies that: for $\lambda \in \operatorname{Int} \mathfrak{a}_{\varepsilon}^{*}$ and $t>0$

$$
\begin{aligned}
\left|\Phi_{\lambda, \delta 1}\left(a_{t}\right)\right|\left|h_{j}^{\delta}(\lambda)\right|(1+|\lambda|)^{m} & \leq c_{1 j} \frac{1}{(1+t)^{n}} \varphi_{0}^{r_{\varepsilon}}\left(a_{t}\right) \\
& \leq c_{1 j}\left(r_{\varepsilon}, t\right), \text { where } c_{1 j}\left(r_{\varepsilon}, t\right)= \begin{cases}c_{1 j} & \text { if } r_{\varepsilon} \geq 0 \\
c_{1 j} e^{\left|r_{\varepsilon}\right| t} & \text { if } r_{\varepsilon}<0\end{cases}
\end{aligned}
$$

The last line of the inequality (4.34) is a consequence of the fact that $\varphi_{0}\left(a_{t}\right)<1$ for all $t>0$ and the two-sided estimate $(2.11)$ of $\varphi_{0}\left(a_{t}\right)$.

Now we express $\Phi_{\lambda, \delta}{ }_{1}\left(a_{t}\right)$ in terms of the Jacobi function (4.21), which reduces (4.34) to the following:

$$
\left|h_{j}^{\delta}(\lambda)\left\|\varphi_{\lambda}^{\alpha+r, \beta+s}(t)\right\| Q_{\delta}(i \lambda+1)(\sinh t)^{r}(\cosh t)^{s}\right|(1+|\lambda|)^{m} \leq \frac{1}{\left\|v_{1}\right\|} c_{1 j}\left(r_{\varepsilon}, t\right) .
$$

We note that $h^{\delta} g^{\delta}(\lambda) Q_{\delta}(1-i \lambda), \lambda \in \operatorname{Int} \mathfrak{a}_{\varepsilon}^{*}$. Hence we get the inequality.

$$
\left|g_{j}^{\delta}(\lambda)\left\|\varphi_{\lambda}^{\alpha+r, \beta+s}(t)\right\| Q_{\delta}(1-i \lambda) Q_{\delta}(i \lambda+1)(\sinh t)^{r} \|(\cosh t)^{s}\right|(1+|\lambda|)^{m} \leq \frac{1}{\left\|v_{1}\right\|} c_{1 j}\left(r_{\varepsilon}, t\right)
$$

We now let $t=\frac{1}{|\lambda|^{2}}$. We choose one $\Lambda>\frac{2}{\pi}$ large enough so that the disk $B^{\Lambda}(0)=\{\lambda|| \lambda \mid \leq \Lambda\}$ contains

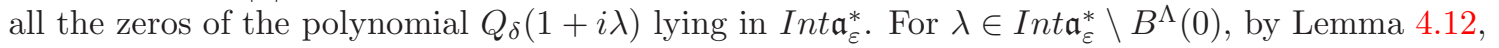

$$
\left|\varphi_{\lambda}^{\alpha+r, \beta+s}\left(\frac{1}{|\lambda|^{2}}\right)\right|>C_{\Lambda, r, s}>0
$$

We note that the polynomial $Q_{\delta}(1+i \lambda) Q_{\delta}(1-i \lambda)$ is of degree $2 r$ (see (2.29)). Hence for all $\lambda \in$ $\operatorname{Inta}_{\varepsilon}^{*} \backslash B^{\Lambda}(0)$ one can find a positive constant $\mathfrak{d}$ such that $\left|Q_{\delta}(1+i \lambda) Q_{\delta}(1-i \lambda)\left(\sinh \frac{1}{\left.\lambda\right|^{2}}\right)^{r}\right|>\mathfrak{d}$.

Also, for the above choice of $\lambda, e^{\left|r_{\varepsilon}\right| \frac{1}{|\lambda|^{2}}} \leq e^{\left|r_{\varepsilon}\right| \frac{1}{|\Lambda|^{2}}}$. Hence from (4.36) we conclude that: for all $\lambda \in \operatorname{Inta}_{\varepsilon}^{*} \backslash B^{\Lambda}(0)$

$$
\left|g_{j}^{\delta}(\lambda)\right|(1+|\lambda|)^{m} \leq \frac{e^{\left|r_{\varepsilon}\right| \frac{1}{|\Lambda|^{2}}}}{C_{\Lambda, \alpha+r, \beta+s} \mathfrak{d}}=c_{\delta}(\text { say }) \text { for each } m \in \mathbb{Z}^{+} .
$$

As $B^{\Lambda}(0)$ contains all the zeros of the polynomial $Q_{\delta}(1-i \lambda)$, so there exists a constant $k_{\delta}>0$ such that $\left|Q_{\delta}(1-i \lambda)\right|>k_{\delta}$ for all $\lambda \in \operatorname{Int} \mathfrak{a}_{\varepsilon}^{*} \backslash B^{\Lambda}(0)$. Thus, for $\lambda \in \operatorname{Int} \mathfrak{a}_{\varepsilon}^{*} \backslash B^{\Lambda}(0)$

$$
\left|h_{j}^{\delta}(\lambda)\right|(1+|\lambda|)^{m}=\frac{1}{\left|Q_{\delta}(1-i \lambda)\right|}\left|g_{j}^{\delta}(\lambda)\right|(1+|\lambda|)^{m} \leq \frac{c_{\delta}}{k_{\delta}} .
$$

For each $1 \leq j \leq d_{\delta}$ the following inequality is also obtained from (4.5): for all $a_{t} \in A^{+}$and $\lambda \in \operatorname{Inta} *$

$$
\left|\left(\frac{d}{d \lambda}\right)\left\{\Phi_{\lambda, \delta 1}\left(a_{t}\right) Q_{\delta}(1-i \lambda) g_{j}^{\delta}(\lambda)\right\}\right|(1+t)^{n}(1+|\lambda|)^{m} \varphi_{0}^{-r_{\varepsilon}}\left(a_{t}\right) \leq c_{1 j}^{\prime} .
$$

That is

$$
\left|\left\{\frac{d}{d \lambda} g_{j}^{\delta}(\lambda)\right\} Q_{\delta}(1-i \lambda) \Phi_{\lambda, \delta 1}\left(a_{t}\right)+g_{j}^{\delta}(\lambda)\left(\frac{d}{d \lambda}\right)\left\{Q_{\delta}(1-i \lambda) \Phi_{\lambda, \delta 1}\left(a_{t}\right)\right\}\right|(1+|\lambda|)^{m} \leq c_{1 j}^{\prime}\left(r_{\varepsilon}, t\right) .
$$

The last line can be written as

$$
\begin{aligned}
& \left|\left\{\frac{d}{d \lambda} g_{j}^{\delta}(\lambda)\right\} Q_{\delta}(1-i \lambda) \Phi_{\lambda, \delta 1}\left(a_{t}\right)\right|(1+|\lambda|)^{m} \\
& \quad \leq c_{1 j}^{\prime}\left(r_{\varepsilon}, t\right)+\left|g_{j}^{\delta}(\lambda)\left(\frac{d}{d \lambda}\right)\left\{Q_{\delta}(1-i \lambda) \Phi_{\lambda, \delta 1}\left(a_{t}\right)\right\}\right|(1+|\lambda|)^{m},
\end{aligned}
$$

writing $\Phi_{\lambda, \delta 1}\left(a_{t}\right)$ in terms of the Jacobi functions as in (4.21) we get,

$$
\begin{array}{r}
\leq c_{1 j}^{\prime}\left(r_{\varepsilon}, t\right)+\frac{(1+|\lambda|)^{m}}{(1+\alpha)_{r}}\left|g_{j}^{\delta}(\lambda)\left(\frac{d}{d \lambda}\right)\left\{Q_{\delta}(1-i \lambda) Q_{\delta}(1+i \lambda)(\sinh t)^{r}(\cosh t)^{s} \varphi_{\lambda}^{\alpha+r, \beta+s}(t)\right\}\right| \\
\leq c_{1 j}^{\prime}\left(r_{\varepsilon}, t\right)+\frac{(1+|\lambda|)^{m}}{(1+\alpha)_{r}}\left|g_{j}^{\delta}(\lambda)\left\{\left(\frac{d}{d \lambda}\right) Q_{\delta}(1-i \lambda) Q_{\delta}(1+i \lambda)\right\}(\sinh t)^{r}(\cosh t)^{s} \varphi_{\lambda}^{\alpha+r, \beta+s}(t)\right|+ \\
\frac{(1+|\lambda|)^{m}}{(1+\alpha)_{r}}\left|g_{j}^{\delta}(\lambda) Q_{\delta}(1-i \lambda) Q_{\delta}(1+i \lambda)(\sinh t)^{r}(\cosh t)^{s}\left\{\left(\frac{d}{d \lambda}\right) \varphi_{\lambda}^{\alpha+r, \beta+s}(t)\right\}\right| .
\end{array}
$$


We rewrite the inequality as

$$
\begin{array}{r}
\left|\left(\frac{d}{d \lambda}\right) g_{j}^{\delta}(\lambda)\right|(1+|\lambda|)^{m}<c_{1 j}^{\prime}\left(r_{\varepsilon}, t\right)\left[\left|Q_{\delta}(1+i \lambda) Q_{\delta}(1-i \lambda)(\sinh t)^{r}\right|(\cosh t)^{s}\left|\varphi_{\lambda}^{\alpha+r, \beta+s}(t)\right|\right]^{-1} \\
+\left|g_{j}^{\delta}(\lambda)\right|(1+|\lambda|)^{m} \frac{\mid \frac{d}{d \lambda}\left(Q_{\delta}(1-i \lambda) Q_{\delta}(1+i \lambda) \mid\right.}{\left|Q_{\delta}(1+i \lambda) Q_{\delta}(1-i \lambda)\right|} \\
+\left|g_{j}^{\delta}(\lambda)\right|(1+|\lambda|)^{m} \frac{\left|\left(\frac{d}{d \lambda}\right) \varphi_{\lambda}^{\alpha+r, \beta+s}(t)\right|}{\left|\varphi_{\lambda}^{\alpha+r, \beta+s}(t)\right|} .
\end{array}
$$

Again we take $t=\frac{1}{|\lambda|^{2}}$ and choose $\Lambda>\frac{2}{\pi}$ suitably large so that $B^{\Lambda}(0)$ contains all the zeros of the polynomial $Q_{\delta}(1-i \lambda)$. We will presently obtain bounds for the three terms on the right-hand side separately for $\lambda \in \operatorname{Int}_{\varepsilon} \mathfrak{a}_{\varepsilon} \backslash B^{\Lambda}(0)$. The first term is bounded by a constant, as seen earlier. We have already obtained a bound for the factor $\left|g_{j}^{\delta}(\lambda)\right|(1+|\lambda|)^{m}$ present in the last two terms. The quotient in the second term is clearly bounded in the region $\operatorname{Inta}_{\varepsilon}^{*} \backslash B^{\Lambda}(0)$. Finally, a bound for the quotient in the last term is obtained from the following facts $\left|\varphi_{\lambda}^{\alpha+r, \tilde{\beta}+s}\left(\frac{1}{|\lambda|^{2}}\right)\right| \geq C_{\Lambda, \alpha+r, \beta+s}$ ( by Lemma 4.12) and $\left|\left(\frac{d}{d \lambda}\right) \varphi_{\lambda}^{\alpha+r, \beta+s}\left(\frac{1}{|\lambda|^{2}}\right)\right| \leq c \frac{1}{|\Lambda|^{2}} e^{(\varepsilon+\varrho)\left(\frac{1}{|\Lambda|^{2}}\right)}$ (by $\left.(4.23)\right)$.

As $h_{j}^{\delta}(\lambda)=Q_{\delta}(1-i \lambda) g_{j}^{\delta}(\lambda)$, so for each $m \in \mathbb{Z}^{+}$

$$
\sup _{\lambda \in \operatorname{Inta} \mathfrak{a}_{\varepsilon}^{*}}\left|\left(\frac{d}{d \lambda}\right) h_{j}^{\delta}(\lambda)\right|(1+|\lambda|)^{m} \leq C_{\delta}<+\infty .
$$

For any order of the derivative on $\lambda$, we can use essentially the same argument.

Corollary 4.15. From the decay (4.32) of each matrix entry of the function $h^{\delta}$ obtained in the above Proposition 4.14 we get: for each fixed $\delta \in \Gamma(f)$, for each $m, n \in \mathbb{Z}^{+} \cup\{0\}$

$$
\sup _{\lambda \in \operatorname{Inta} \mathfrak{a}_{\varepsilon}^{*}}\left\|\left(\frac{d}{d \lambda}\right)^{m} h^{\delta}(\lambda)\right\|_{2}(1+|\lambda|)^{n}<+\infty .
$$

Let us now recollect the properties we have obtained for the function $h^{\delta}\left(\delta \in \widehat{K}_{M}\right)$ in the following Lemma.

Lemma 4.16. The function $h^{\delta}$ (as obtained in (4.12)) is a $\operatorname{Hom}\left(V_{\delta}, V_{\delta}^{M}\right)$ valued function on $\mathfrak{a}_{\varepsilon}^{*}$ which satisfies the following properties:

(i) $h^{\delta}$ is holomorphic in interior of $\mathfrak{a}_{\varepsilon}^{*}$ and it extends to the closed strip $\mathfrak{a}_{\varepsilon}^{*}$ as a continuous function.

(ii) $\lambda \mapsto Q_{\delta}(1-i \lambda)^{-1} h^{\delta}(\lambda)$ is an even function and also it is holomorphic on Inta $\mathfrak{a}_{\varepsilon}^{*}$.

(iii) for each $m, n \in \mathbb{Z}^{+} \cup\{0\}$

$$
\sup _{\lambda \in \operatorname{Inta}_{\varepsilon}^{*}}\left\|\left(\frac{d}{d \lambda}\right)^{m} h^{\delta}(\lambda)\right\|_{\mathbf{2}}(1+|\lambda|)^{n}<+\infty
$$

The above Lemma shows that for each $\delta \in \widehat{K}_{M}, h^{\delta} \in \mathcal{S}_{\delta}\left(\mathfrak{a}_{\varepsilon}^{*}\right)$ (see Definition 2.5 ). Hence by Theorem 2.6 the inversion $\mathrm{J} h \delta$ given by

$$
J^{\delta}(x)=\int_{\mathfrak{a}^{*+}} \Phi_{\lambda, \delta}(x) h^{\delta}(\lambda)|\mathbf{c}(\lambda)|^{-2} d \lambda,
$$

belongs to the $p$ th Schwartz class $\mathcal{S}^{p}(X)$ where $p$ is determined by the chosen $\varepsilon$ by $p=\frac{2}{1+\varepsilon}$.

It is clear from (4.45), (4.12) and (4.2) that $f^{\delta} \equiv \sqrt{d_{\delta}} \mathcal{J} h^{\delta}$. Hence for each $\delta$, the function $\operatorname{trf} f^{\delta}$ satisfies the Schwartz space decay condition: for each $\mathbf{D}, \mathbf{E} \in \mathcal{U}(\mathfrak{g})$ and $n \in \mathbb{Z}^{+} \cup\{0\}$,

$$
\sup _{x \in X}|\operatorname{trf} \delta(\mathbf{D} ; x ; \mathbf{E})|(1+|x|)^{n} \varphi_{0}^{-\frac{2}{p}}(x) \leq+\infty .
$$

The function $f$ obtained in (4.2) was proved to be left- $K$-finite. Hence $f \in \mathcal{S}^{p}(X)$ where $p=\frac{2}{1+\varepsilon}$. 
Finally we shall show that $P_{\lambda} f(x)=f_{\lambda}(x)\left(\lambda \in \mathfrak{a}_{\varepsilon}^{*}\right)$ where the function $f$ is obtained in (4.2). As $f$ is left $K$ finite $P_{\lambda} f$ can be decomposed as follows

$$
\begin{aligned}
P_{\lambda} f(x) & =\sum_{\delta \in \Gamma(f)} \operatorname{tr}\left(P_{\lambda} f\right)^{\delta}(x) \\
& =\sum_{\delta \in \Gamma(f)} \operatorname{tr} P_{\lambda}(f)^{\delta}(x),
\end{aligned}
$$

where the last line follows by using Proposition 3.1, and $\Gamma(f)$ being a finite subset of $\widehat{K}_{M}$. Now from the above discussion and Proposition 3.1 we get the following:

$$
P_{\lambda}(f)^{\delta}(x)=\Phi_{\lambda, \delta}(x) \widetilde{f^{\delta}}(\lambda)=\Phi_{\lambda, \delta}(x) h^{\delta}(\lambda)=f_{\lambda}^{\delta}(x), \quad \lambda \in \mathfrak{a}_{\varepsilon}^{*}
$$

Thus (4.47) can be reformulated as $P_{\lambda} f(x)=\sum_{\delta \in \Gamma(f)} \operatorname{tr} f_{\lambda}^{\delta}(x)$, which immediately gives $P_{\lambda} f(x)=f_{\lambda}(x)$ for all $\lambda \in \mathfrak{a}_{\varepsilon}^{*}$ and $x \in X$. We give the gist of what we have shown in this section in the form of the following theorem

Theorem 4.17. Any continuous function $g:(x, \lambda) \mapsto g_{\lambda}(x)$ defined on $X \times \mathfrak{a}_{\varepsilon}^{*}$ with certain $\varepsilon \geq 0$ and satisfying the conditions of Definition 4.1 is of the form $g_{\lambda}(x)=P_{\lambda} f(x)$ for some left-K-finite function $f \in \mathcal{S}^{p}(X)$ where $p=\frac{2}{1+\varepsilon}$.

\section{Inverse Paley-Wiener Theorem}

We begin the section with a definition.

Definition 5.1. Let $P_{R}(X)$ be the class of scalar valued functions $(\lambda, x) \mapsto f_{\lambda}(x)$ on $\mathfrak{a}^{*} \times X$ satisfying:

(i) the map $\lambda \mapsto f_{\lambda}(\cdot)$ is an even, compactly supported $\mathrm{e}^{\infty}$ function on $\mathfrak{a}^{*}$ with it support lying in $[-R, R]$ (note that our group $G$ is of real rank-one and thus we have identified $\mathfrak{a}^{*}$ with $\mathbb{R}$ ),

(ii) for each $\lambda \in \mathfrak{a}^{*}, x \mapsto f_{\lambda}(x)$ is a $C^{\infty}$ function on $X$ and $f_{\lambda}(\cdot) \in \mathcal{E}_{\lambda}(X)$.

In this section we shall try to characterize the space $P_{R}(X)$ as an image of certain subspace of $L^{2}(X)$ under the spectral projection.

We need to recall some basic results regarding a certain $G$-invariant domain $\Xi$ in $G_{\mathbb{C}} / K_{\mathbb{C}}$ called the complex crown. Here $X_{\mathbb{C}}=G_{\mathbb{C}} / K_{\mathbb{C}}$ is the natural complexification (see [KÓS05]) of the symmetric space $X$. The domain can be explicitly written as $\Xi=G \exp i \Omega \cdot x_{0}$, where $x_{0}=e K$ and $\Omega=\{H \in$ $\left.\mathfrak{a}|| \alpha(H) \mid<\frac{\pi}{2}, \alpha \in \Sigma\right\}[\mathrm{KS} 05]$. Let $\mathcal{G}(\Xi)$ be space of all holomorphic functions on the complex crown. For $\lambda \in i \mathfrak{a}^{*}$, the function $H \mapsto \varphi_{\lambda}(\exp i H)(H \in \mathfrak{a})$ can be analytically continued in the tube domain $\mathfrak{a}+2 i \Omega[\mathrm{KS} 04]$. Almost all the basic analysis on the crown domain uses a fundamental tool called the orbital integrals developed by Gindikin et al. [GK02]. Let $h$ be a function on $\Xi$ suitably decreasing at the boundary and $Y \in 2 \Omega$, then the orbital integral is defined by

$$
O_{h}(i Y)=\int_{G} h\left(g \exp \left(\frac{i}{2} Y\right) \cdot x_{0}\right) d g .
$$

If a holomorphic function $\theta$ on the tube $\mathfrak{a}+2 i \Omega$ has the representation

$$
\theta(Y)=\int_{i \mathfrak{a}^{*}} g(\lambda) \varphi_{\lambda}(\exp Y)|\mathbf{c}(\lambda)|^{-2} d \lambda
$$

then we define (with certain condition on $g$, see [KÓS05]) the operator

$$
D \theta(Y)=\int_{i \mathfrak{a}^{*}} g(\lambda) \psi_{\lambda}(Y)|\mathbf{c}(\lambda)|^{-2} d \lambda,
$$

where $\psi_{\lambda}(Y)=e^{\langle\lambda, Y\rangle}+e^{\langle\lambda,-Y\rangle}$. The operator $D$ is a pseudo-differential shift operator [KÓS05]. The following is an inverse Paley-Wiener theorem for the Helgason Fourier transform.

Theorem 5.2. [Thangavelu [Tha07, Theorem 2.3]]

Let $f \in L^{2}(X)$, then the Helgason Fourier transform $\mathcal{F} f(\lambda, k M)$ is supported in $|\lambda| \leq R$ if and only if the function $f$ has a holomorphic extension $F \in \mathcal{G}(\Xi)$ which satisfies the estimate

$$
D O_{|F|^{2}}(i Y) \leq C e^{2 R|Y|} \text {, where } C \text { is independent of } Y \in 2 \Omega \text {. }
$$


Our main theorem in this section is a consequence of the above theorem.

Theorem 5.3. A function $f_{\lambda}(x)\left(x \in X, \lambda \in \mathfrak{a}^{*}\right)$ is in $P_{R}(X)$ if and only if $f_{\lambda}(x)=\left(f * \varphi_{\lambda}\right)(x)$ $\left(\forall x \in X, \lambda \in \mathfrak{a}^{*}\right)$ for some $f \in L^{2}(X)$ which admits a holomorphic extension $F \in \mathcal{G}(\Xi)$ satisfying the estimate (5.2)

Proof. Let $f_{\lambda}(x) \in P_{R}(X)$; then we get a function

$$
f(x)=\int_{\mathfrak{a}^{*+}} f_{\lambda}(x)|\mathbf{c}(\lambda)|^{-2} d \lambda .
$$

The integral (5.3) is obviously convergent and $f \in \mathrm{C}^{\infty}(X)$. A simple application of the Peter-Weyl theorem gives

$$
f(x)=\int_{\mathfrak{a}^{*+}}\left[\sum_{\delta \in \widehat{K}_{M}} \operatorname{tr} f_{\lambda}^{\delta}(x)\right]|\mathbf{c}(\lambda)|^{-2} d \lambda .
$$

Now for each $\delta \in \widehat{K}_{M}$ and $\lambda \in \mathfrak{a}^{*}, f_{\lambda}^{\delta} \in \mathcal{E}_{\lambda}^{\delta}(X)$, hence by Theorem 4.12 we can write $f_{\lambda}^{\delta}(x)=$ $\sqrt{d_{\delta}} \Phi_{\lambda, \delta}(x) h^{\delta}(\lambda)$. As $\lambda \mapsto f_{\lambda}^{\delta}$ is compactly supported and $\Phi_{\lambda, \delta}$ is an entire function so the function $h^{\delta}$ must have its support in $[-R, R]$. The above structural form can further reduce (5.4) as follows:

$$
\begin{aligned}
f(x) & =\int_{\mathfrak{a}^{*+}}\left[\sum_{\delta \in \widehat{K}_{M}} \sqrt{d_{\delta}} \sum_{i=1}^{d_{\delta}}\left\langle\Phi_{\lambda, \delta}(x) h^{\delta}(\lambda) v_{i}, v_{i}\right\rangle\right]|\mathbf{c}(\lambda)|^{-2} d \lambda \\
& =\int_{\mathfrak{a}^{*+}}\left[\sum_{\delta \in \widehat{K}_{M}} \sqrt{d_{\delta}} \sum_{i=1}^{d_{\delta}}\left\langle\Phi_{\lambda, \delta}(x) v_{1}, v_{i}\right\rangle\left\langle h^{\delta}(\lambda) v_{i}, v_{1}\right\rangle\right]|\mathbf{c}(\lambda)|^{-2} d \lambda \\
& =\int_{\mathfrak{a}^{*+}}\left[\sum_{\delta \in \widehat{K}_{M}} \sqrt{d_{\delta}} \sum_{i=1}^{d_{\delta}}\left\langle\int_{K} e^{-(i \lambda+1) H\left(x^{-1} k\right)} \delta(k) d k v_{1}, v_{i}\right\rangle\left\langle h^{\delta}(\lambda) v_{i}, v_{1}\right\rangle\right]|\mathbf{c}(\lambda)|^{-2} d \lambda \\
& =\int_{\mathfrak{a}^{*+}} \int_{K} e^{-(i \lambda+1) H\left(x^{-1} k\right)}\left[\sum_{\delta \in \widehat{K}_{M}} \sqrt{d_{\delta}} \sum_{i=1}^{d_{\delta}}\left\langle\delta(k) v_{1}, v_{i}\right\rangle\left\langle h^{\delta}(\lambda) v_{i}, v_{1}\right\rangle\right]|\mathbf{c}(\lambda)|^{-2} d k d \lambda \\
& =\int_{\mathfrak{a}^{*+}} \int_{K} e^{-(i \lambda+1) H\left(x^{-1} k\right)}\left[\sum_{\delta \in \widehat{K}_{M}} \sqrt{d_{\delta}} \operatorname{tr}\left(\delta(k) h^{\delta}(\lambda)\right)\right]|\mathbf{c}(\lambda)|^{-2} d k d \lambda .
\end{aligned}
$$

We denote the function $h(\lambda, k)=\sum_{\delta \in \widehat{K}_{M}} \sqrt{d_{\delta}} \operatorname{tr}\left(\delta(k) h^{\delta}(\lambda)\right)$ in (5.5). Then clearly it is a $\mathrm{C}^{\infty}$ function in the $\lambda$ variable and the function $\lambda \mapsto \int_{K} e^{(-i \lambda+1) H\left(x^{-1} k\right)} h(\lambda, k) d k$ is even. Also $h(\lambda, \cdot)$ is a compactly supported function and $f$ is nothing but the Helgason Fourier inversion of the function $h$. Hence by [Tha07, Theorem 2.3], the function $f \in L^{2}(X)$ and it admits a holomorphic extension on the complex crown satisfying the estimate (5.2).

On the other hand if $g \in L^{2}(X)$ then, for all $\lambda \in \mathfrak{a}^{*}$ and $x \in X$,

$$
P_{\lambda} g(x)=g * \varphi_{\lambda}(x)=\int_{K} e^{-(i \lambda+1) H\left(x^{-1} k\right)} \widetilde{g}(\lambda, k) d k .
$$

Furthermore if $g$ can be extended holomorphically to some $\bar{g} \in \mathcal{G}(\Xi)$ with $\bar{g}$ satisfying (5.2) then by Theorem $5.2, \widetilde{g}(\lambda, \cdot)$ is supported in $[-R, R]$. It is now easy to show that $g * \varphi_{\lambda}(\cdot) \in P_{R}(X)$.

\section{REFERENCES}

[Ank87] Jean-Philippe Anker, La forme exacte de l'estimation fondamentale de Harish-Chandra, C. R. Acad. Sci. Paris Sér. I Math. 305 (1987), no. 9, 371-374.

[Ank91] The spherical Fourier transform of rapidly decreasing functions. A simple proof of a characterization due to Harish-Chandra, Helgason, Trombi, and Varadarajan, J. Funct. Anal. 96 (1991), no. 2, 331-349.

[Ank92] Sharp estimates for some functions of the Laplacian on noncompact symmetric spaces, Duke Math. J. 65 (1992), no. 2, 257-297. 
[Bra96] William O. Bray, Generalized spectral projections on symmetric spaces of noncompact type: Paley-Wiener theorems, J. Funct. Anal. 135 (1996), no. 1, 206-232.

[EK76] Masaaki Eguchi and Atsutaka Kowata, On the Fourier transform of rapidly decreasing functions of $L^{p}$ type on a symmetric space, Hiroshima Math. J. 6 (1976), no. 1, 143-158.

[GK02] Simon Gindikin and Bernhard Krötz, Invariant Stein domains in Stein symmetric spaces and a nonlinear complex convexity theorem, Int. Math. Res. Not. (2002), no. 18, 959-971.

[GKÓ06a] Simon Gindikin, Bernhard Krötz, and Gestur Ólafsson, Holomorphic horospherical transform on noncompactly causal spaces, Int. Math. Res. Not. (2006), Art. ID 76857, 47.

[GKÓ06b] - Horospherical model for holomorphic discrete series and horospherical Cauchy transform, Compos. Math. 142 (2006), no. 4, 983-1008.

[GV88] Ramesh Gangolli and V. S. Varadarajan, Harmonic analysis of spherical functions on real reductive groups, Ergebnisse der Mathematik und ihrer Grenzgebiete [Results in Mathematics and Related Areas], vol. 101, Springer-Verlag, Berlin, 1988.

[HC76] Harish-Chandra, Harmonic analysis on real reductive groups. II. Wavepackets in the Schwartz space, Invent. Math. 36 (1976), 1-55.

[Hel70] Sigurdur Helgason, A duality for symmetric spaces with applications to group representations, Advances in Math. 5 (1970), 1-154 (1970).

[Hel74] Eigenspaces of the Laplacian; integral representations and irreducibility, J. Functional Analysis 17 (1974), 328-353.

[Hel87] - Some results on Radon transforms, Huygens' principle and X-ray transforms, Integral geometry (Brunswick, Maine, 1984), Contemp. Math., vol. 63, Amer. Math. Soc., Providence, RI, 1987, pp. 151-177.

[Hel94] Geometric analysis on symmetric spaces, Mathematical Surveys and Monographs, vol. 39, American Mathematical Society, Providence, RI, 1994.

[Hel00] Groups and geometric analysis, Mathematical Surveys and Monographs, vol. 83, American Mathematical Society, Providence, RI, 2000, Integral geometry, invariant differential operators, and spherical functions, Corrected reprint of the 1984 original.

[Ion00] Alexandru D. Ionescu, On the Poisson transform on symmetric spaces of real rank one, J. Funct. Anal. 174 (2000), no. 2, 513-523.

[JS07] Joydip Jana and Rudra P. Sarkar, On the schwartz space isomorphism theorem for the rank-1 symmetric spaces, Proc. Indian Acad. Sci.(Math. Sci.) 117 (2007), no. 3, 333-348.

[Koo84] Tom H. Koornwinder, Jacobi functions and analysis on noncompact semisimple Lie groups, Special functions: group theoretical aspects and applications, Math. Appl., Reidel, Dordrecht, 1984, pp. 1-85.

[Kos69] Bertram Kostant, On the existence and irreducibility of certain series of representations, Bull. Amer. Math. Soc. 75 (1969), 627-642.

[KÓS05] Bernhard Krötz, Gestur Ólafsson, and Robert J. Stanton, The image of the heat kernel transform on Riemannian symmetric spaces of the noncompact type, Int. Math. Res. Not. (2005), no. 22, 1307-1329.

[KS04] Bernhard Krötz and Robert J. Stanton, Holomorphic extensions of representations. I. Automorphic functions, Ann. of Math. (2) 159 (2004), no. 2, 641-724.

[KS05] B. Krötz and R. J. Stanton, Holomorphic extensions of representations. II. Geometry and harmonic analysis, Geom. Funct. Anal. 15 (2005), no. 1, 190-245.

[Str88] Robert S. Strichartz, Local harmonic analysis on spheres, J. Funct. Anal. 77 (1988), no. 2, 403-433.

[Str89] - Harmonic analysis as spectral theory of Laplacians, J. Funct. Anal. 87 (1989), no. 1, 51-148.

[Str91] $\quad L^{p}$ harmonic analysis and Radon transforms on the Heisenberg group, J. Funct. Anal. 96 (1991), no. 2, 350-406.

[Str92] Corrigendum to: "Harmonic analysis as spectral theory of Laplacians" [J. Funct. Anal. 87 (1989), no. 1, 51-148; MR1025883 (91c:43015)], J. Funct. Anal. 109 (1992), no. 2, 457-460.

[Sug71] Mitsuo Sugiura, Fourier series of smooth functions on compact Lie groups, Osaka J. Math. 8 (1971), 33-47.

[Tha07] S. Thangavelu, A Paley-Wiener theorem for the inverse Fourier transform on some homogeneous spaces, Hiroshima Math. J. 37 (2007), no. 2, 145-159.

Department of Mathematics, Syamaprasad College; 92, S. P. Mukherjee Road; Kolkata- 700026

E-mail address: joydipjana@gmail.com 